OPEN ACCESS

Edited by:

Linlin Wang,

Zhejiang University, China

Reviewed by:

Kun Xiong,

Independent researcher, Changsha,

China

${ }^{*}$ Correspondence:

Junsong Wu

wjs1288@zju.edu.cn

tThese authors have contributed equally to this work

Specialty section:

This article was submitted to

Cell Death and Survival,

a section of the journal

Frontiers in Cell and Developmental

Biology

Received: 24 November 2021

Accepted: 03 January 2022

Published: 02 February 2022

Citation:

Zhou C, Zheng J, Fan $Y$ and Wu J (2022) TI: NLRP3 InflammasomeDependent Pyroptosis in CNS Trauma:

A Potential Therapeutic Target.

Front. Cell Dev. Biol. 10:821225.

doi: 10.3389/fcell.2022.821225

\section{TI: NLRP3 Inflammasome-Dependent Pyroptosis in CNS Trauma: A Potential Therapeutic Target}

\author{
Conghui Zhou ${ }^{1 \dagger}$, Jinfeng Zheng ${ }^{1 \dagger}$, Yunpeng Fan ${ }^{1}$ and Junsong $\mathrm{Wu}^{2 *}$ \\ ${ }^{1}$ The First Affiliated Hospital of Zhejiang University, School of Medicine, Zhejiang University, Hangzhou, China, ${ }^{2}$ Department of \\ Orthopaedics of the First Affiliated Hospital, School of Medicine, Zhejiang University, Hangzhou, China
}

Central nervous system (CNS) trauma, including traumatic brain injury (TBI) and traumatic spinal cord injury (SCI), is characterized by high morbidity, disability, and mortality. TBI and $\mathrm{SCI}$ have similar pathophysiological mechanisms and are often accompanied by serious inflammatory responses. Pyroptosis, an inflammation-dependent programmed cell death, is becoming a major problem in CNS post-traumatic injury. Notably, the pyrin domain containing 3 (NLRP3) inflammasome is a key protein in the pyroptosis signaling pathway. Therefore, underlying mechanism of the NLRP3 inflammasome in the development of CNS trauma has attracted much attention. In this review, we briefly summarize the molecular mechanisms of NLRP3 inflammasome in pyroptosis signaling pathway, including its prime and activation. Moreover, the dynamic expression pattern, and roles of the NLRP3 inflammasome in CNS post-traumatic injury are summarized. The therapeutic applications of NLRP3 inflammasome activation inhibitors are also discussed.

Keywords: NLRP3, pyroptosis, spinal cord injury, traumatic brain injury, CNS trauma

\section{INTRODUCTION}

Central nervous system (CNS) traumatic diseases, including spinal cord injury (SCI) and traumatic brain injury (TBI), are characterized by a high disability rate and various complications, and some hypotheses propose that high disability and mortality rates occur because of secondary injury development. Surgical treatment remains an effective clinical method. Olfactory ensheathing cell transplantation, hematopoietic stem cell transplantation, hematopoietic progenitor cell transplantation, novel cell transdifferentiation techniques, or combined with scaffold therapy have presented new possibilities for nerve repair (Bryukhovetskiy, 2018; Ma et al., 2019; Kuang et al., 2021). Additionally, SCI and TBI share common pathological features, and their complex pathophysiological processes can be classified as primary and secondary injuries. Primary injury is caused by direct external force to the spinal cord or brain, and the damage caused at this stage is irreversible. Delayed secondary injury cascade involves several complex pathophysiological events, including glutamate excitotoxicity, oxidative stress, and inflammatory response (Toklu et al., 2009; Zendedel et al., 2016). Glutamate over-release stimulates glutamate receptors, leading to persistent excitatory toxicity, cell dysfunction, and death after SCI and TBI, and $\mathrm{Na}^{+}$-dependent glutamate transporters GLT-1 and GLAST actively transport excess glutamate, although downregulated after injury (Yi and Hazell, 2006; Fernandez-Lopez et al., 2014). Furthermore, glutamate-induced stimulation is mediated by $\mathrm{Na}^{+} / \mathrm{K}^{+}$-ATPase in addition to $\mathrm{Na}^{+} /$glutamate cotransporter (Pellerin and Magistretti, 1994). $\mathrm{Na}^{+} / \mathrm{K}^{+}$-ATPase dysfunction is closely related to SCI and TBI and can indirectly reflect the severity of the injury. The $\mathrm{Na}^{+} / \mathrm{K}^{+}$-ATPase pump, in particular, is highly sensitive to oxidative stress (Lima et al., 2008; Boon et al., 2012). 
As a response of the innate immune system in the CNS, neuroinflammation plays a key role in the second stage of TBI and SCI, of which the most important step is the activation of the various inflammasome complexes (Zhou et al., 2016). Additionally, the occurrence of neuroinflammation not only represents damage to the brain and spinal cord, especially the activation of macrophages and microglia, but is also is known to be an essential protective mechanism (Mortezaee et al., 2018). However, excessive neuroinflammation tends to hinder repair and regeneration, and the NOD-like receptor family, pyrin domain containing 3 (NLRP3) inflammasome-mediated neuroinflammation appears to be responsible for the unfavorable outcome in CNS injury. Activation of the NLRP3 inflammasome is the key to pyroptosis and the trigger of the inflammatory response, which plays an important role in the pathogenesis of various inflammatory diseases (Mangan et al., 2018). Nevertheless, the role of the NLRP3 inflammasome in CNS trauma still remains complex and controversial. Here, we focus on the molecular mechanisms of NLRP3 inflammasome in the pyroptosis signaling pathway, including its prime, activation, and post-translational modifications. Moreover, the dynamic expression pattern, and roles of the NLRP3 inflammasome in CNS trauma are summarized. The pharmacological applications of NLRP3 inflammasome inhibitors in CNS trauma are also discussed.

\section{PYROPTOSIS AND INFLAMMASOMES}

Pyroptosis is a novel inflammatory type of programmed cell death (PCD) that is linked to the activation of inflammasomes (Ousingsawat et al., 2019). Pyroptosis was originally described in 1992 by Zychlinsky et al. (1992), who found that a cell death form of lysis way of infected macrophages caused by Shigella flexneri. It was formerly believed to be apoptosis because it shares some features with apoptosis, including DNA fragmentation, nuclear pyknosis (chromatin condensation), and caspase dependence (Ye et al., 2019). However, it is not identical to apoptosis, especially in terms of its morphological features. Subsequent research detected macrophages infected by various pathogens such as Salmonella typhimurium, Listeria monocytogenes, and Pseudomonas aeruginosa. Caspase-1-dependent cell death releases a large amount of pro-inflammatory factors (Jesenberger et al., 2000; Breitbach et al., 2009; Wu et al., 2010). Caspase-1 plays a significant role in the maturation of pro-inflammatory cytokines. Once triggered, caspase- 1 potentially catalyzes the conversion of inactive precursors interleukin-1 $\beta$ (IL-1 $\beta$ ) and interleukin-18 (IL18 ) into mature bioactive forms, thus magnifying innate and adaptive immune responses (Ippagunta et al., 2011). In 2001, Cookson and Brennan (2001) first used the term "pyroptosis" to define this novel type of caspase-1-dependent regulated cell death, which was used to distinguish it from apoptosis. Pyroptosis is correlated with the progression of various human diseases, including cancer, neurodegenerative disease and cardiovascular disease (Ma et al., 2018). Furthermore, the role of pyroptosis in the development of CNS post-traumatic injury has garnered significant interest in research.
Pyroptosis involves cell swelling, pore formation in the plasma membrane, membrane rupture, significant inflammation, and release of a large amount of cytosolic contents (Shi et al., 2015). There are two pyroptosis signaling pathways: the canonical (caspase-1 dependent) pathway and the noncanonical (caspase-1 independent) pathway (Figure 1). The pyroptosis pathway is inseparable from inflammasomes. The assembly of the inflammasome is a feature of the inflammatory cascade, which is a multiprotein complex expressed in the cytoplasm of the cell and is composed of pattern recognition receptor (PRR) activation (Ferreri et al., 2010). PRRs are involved in recognizing specific patterns of microorganisms and are key to detecting pathogen invasion. PRRs are further classified into five families: Toll-like receptors (TLRs), NOD-like receptors (NLRs), C-type lectin receptors (CTLs), AIM2-like receptors (ALRs), and retinoic acid-inducible gene (RIG)-I-like receptors (RLRs), among which the main ones closely tied with pyroptosis are TLRs, NLRs, and ALRs (Lamkanfi and Dixit, 2014; Marongiu et al., 2019). PRRs interact with certain pathogen-associated molecular patterns (PAMPs) from microorganisms or nonpathogen-related damage-associated molecular patterns (DAMPs) released by host cells under damaged conditions to trigger the innate immune responses (Reed et al., 2013).

Inflammasomes are also divided into canonical and noncanonical inflammasomes (Figure 2). Canonical inflammasomes include double-stranded DNA (dsDNA) sensors absent in melanoma 2 (AIM2), pyrin inflammasomem, and NLRP subfamily (NLRP1, NLRP3, and NLRC4) (He X.-m. et al., 2020). Non-canonical inflammasomes include mouse caspase-11 and human caspase- 4 and caspase-5, and are highly homologous (Yi, 2020). Among the numerous inflammasome complexes, the NLRP3 inflammasome is the most closely related to pyroptosis and is the best-characterized inflammasome. The classic NLRP3 inflammasome consists of three components, namely the NLRP3 receptor, adaptor protein apoptotic speck protein (ASC), and effector caspase-1, of which NLRP3 receptor is the key component and is composed of an $\mathrm{N}$-terminal pyrin domain (PYD), a central nucleotide-binding and oligomerization domain referred to as NACHT and a C-terminal LRR domain. When activated, NLRP3 oligomerizes through homotypic interactions of their NACHT domains. Oligomerized NLRP3 recruits ASC via homotypic PYD-PYD interactions and subsequently recruits caspase-1 through CARD-CARD interactions (Lu et al., 2014; Schmidt et al., 2016; Boucher et al., 2018). NLRP3 inflammasomes are mainly expressed in immune cells (monocytes, dendritic cells, neutrophils, and lymphocytes), epithelial cells, and osteoblasts (Zhong et al., 2013). Moreover, recent studies have shown that NLRP3 inflammasomes are expressed in the microglia and neurons in the brain and spinal cord as well (Chen et al., 2019b; Chen et al., 2019c).

Several reports have shown that NIMA-related kinase 7 (NEK7), a mitotic serine/threonine kinase, is an essential component of NLRP3 inflammasome activation through interaction with NLRP3 (Huang et al., 2018). The two domains of NEK7, the N-lobe and the C-lobe, interact 


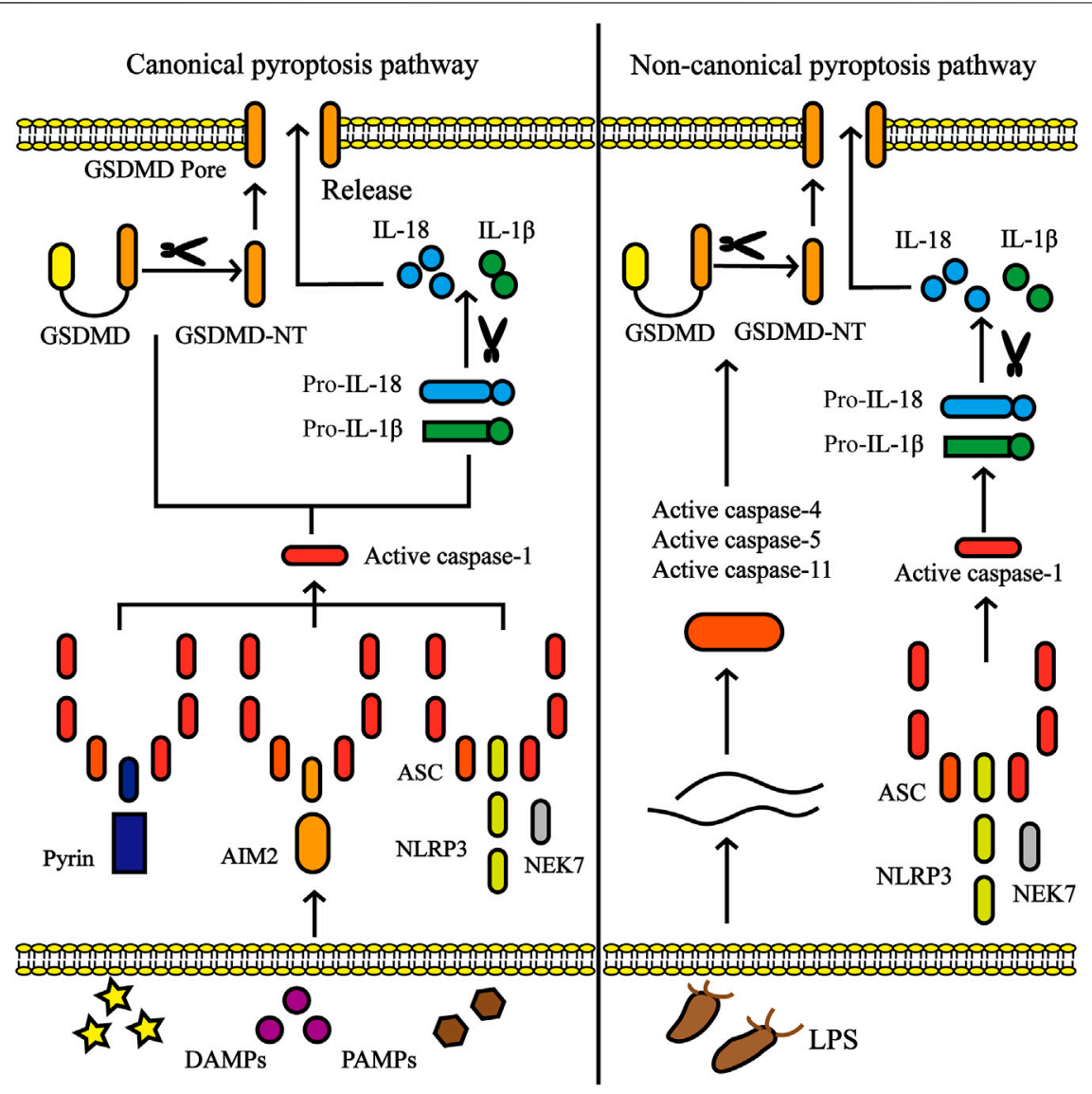

FIGURE 1 | Canonical pyroptosis pathway and non-canonical pyroptosis pathway. In the canonical pyroptosis pathway, activation of inflammasome (including NLRP3, AIM2 or pyrin) occurs via a multitude of pathogen-associated molecular patterns (PAMPs) or damage-associated molecular patterns (DAMPs). Once activated, NLRP3 oligomerizes through homotypic interactions of their NACHT domains. The oligomerized NLRP3 recruits ASC via homotypic PYD-PYD interactions and subsequently recruits caspase- 1 through CARD-CARD interactions. The activated caspase- 1 involves the cleavage of pro-IL-1 $\beta / 18$ into IL- $1 \beta / 18$ and Gasdermin $\mathrm{D}$ (GSDMD) into N-terminal fragment of GSDMD (GSDMD-NT), which forms the pores in the plasma membrane, accompanied by the secretion of IL-1 $\beta / 18$. In noncanonical pyroptosis pathway, caspase-4/5/8/11 are activated by intracellular lipopolysaccharide (LPS) derived by bacteria, leading to pyroptosis by the cleavage of GSDMD.

directly with NLRP3, and NEK7 oligomerizes NLRP3 to form a functional complex, which is closely related to ASC oligomerization and ASC speck formation (Chen X. et al., 2019; Shi et al., 2020). Interestingly, $\mathrm{K}^{+}$efflux is a driver event in upstreaming of NEK7-mediated NLRP3 activation (Chen et al., 2019b). Once NEK7 binds to NLRP3, it triggers a series of caspase cascade and induces pyroptosis. Therefore, NEK7, as an upstream regulator of NLRP3 inflammasome activation, may be a potential therapeutic target.

\section{ROLES OF NLRP3 INFLAMMASOME IN PYROPTOSIS}

Although the underlying activation mechanism of the NLRP3 inflammasome is still not clearly elucidated, two signal activation mechanisms are currently generally accepted. Two signals are required for the NLRP3 inflammasome to function: the priming signal (signal 1) and activation signal (signal 2).

\subsection{Priming of NLRP3 Inflammasome}

The priming signal is a prerequisite for the activation signal, and its main function is to up-regulate the inflammatory component and make it in a state of inactivation but capable of responding to the signal. The priming signal refers to the activation of the NF$\kappa \mathrm{B}$ signaling pathway through PRRs receptors, such as TLRs, NLRs, or cytokine receptors that activate the transcription factor $\mathrm{NF}-\kappa \mathrm{B}$, including tumor necrosis factor (TNF) and interleukin- $1 \beta$ (IL-1 $\beta$ ) (Bauernfeind et al., 2009). Subsequently, activated NF- $\kappa B$ upregulated the expression of inflammation-related genes $N L R P 3$, pro- $I L-1 \beta$, and pro- $I L-18$, which are considered to play a transcriptional upregulatory role (Bauernfeind et al., 2009; Pecorelli et al., 2020). Strikingly, although NF-kB is upstream of NLRP3, overexpression of NLRP3, in turn, activates the NF- $\kappa B$ signaling pathway (Peng et al., 2020). In addition, it has been reported that the initiation of the NLRP3 inflammasome is also affected by FAS-associated death domain protein (FADD) and non-apoptotic caspase-8 (Allam et al., 2014; Gurung et al., 2014). Caspase- 8 deficiency inhibits the activation of caspase- 1 and 




FIGURE 2 | Structures of canonical inflammasomes and non-canonical inflammasomes. (A) Domain organization of canonical inflammasomes. The nucleotidebinding oligomerization domain (NOD)-like receptors (NLRs) have common domains, which include the $\mathrm{C}$-terminal leucine-rich repeat (LRR), NACHT, and the N-terminal effector domains. The black bidirectional arrows represent the interaction between homotype PYD/PYD and CARD/CARD. Double-stranded DNA (dsDNA) sensors absent in melanoma 2 (AIM2) are composed of a C-terminal dsDNA-binding HIN200 domain and an N-terminal PYD. The pyrin protein contains an amino-terminal PYD, a B-box, a coiled-coil, a Spla and Ryanodine receptor (SPRY) domain and a PRY domain. (B) Structures and activation of non-canonical inflammasomes. Procaspase-11 (mouse) and pro-caspase-4/5 (human) are composed of the same structure, namely CARD, P20 and P10. LPS directly interacts with the CARD motif of procaspase-11 or pro-caspase-4/5 through the lipid A moiety of LPS. Activation of caspase-4/5/11 non-canonical inflammasome by oligomerization of the LPS-caspase-4/ $5 / 11$ complex.

caspase-11 by NLPR3 (Gurung et al., 2014). Caspase- 8 not only promotes NLRP3 inflammasome activation and IL-1/IL-18 release but also separates gasdermin $\mathrm{D}$ (GSDMD) into the functional $\mathrm{N}$-terminal fragment of GSDMD (GSDMD-NT) domain that triggers pyroptosis (Orning et al., 2018; Sarhan et al., 2018). Recent studies have reported that cellular FLICEinhibitory protein (c-FLIP) contains a caspase-like domain similar to caspase-8, which can also cleave GSDMD to release the GSDMD-NT (Huang et al., 2021). FADD is responsible for recruiting pro-caspase- 8 and converting it into active caspase- 8 , leading to the initiation of the caspase cascade (Martinet et al., 2019). Among the TLR/IL-1R signaling pathway, TRAF6 mediates non-transcriptional priming of the NLRP3 inflammasome in the presence of ubiquitin E3 ligase activity (Xing et al., 2017). Moreover, as signal molecules downstream of TLRs and MyD88, IL-1 receptor-associated kinase 1 (IRAK-1) and IRAK-4 play an important role in the rapid transcription independent of NLRP3 priming (Fernandes-Alnemri et al., 2013; Lin et al., 2014). At the priming stage, BRCA1/BRCA2-containing complex subunit 3 (BRCC3), a deubiquitinating enzyme, triggers the deubiquitination of the LRR region of NLRP3, promoting the activation of the NLRP3 inflammasome (Juliana et al., 2012; Py et al., 2013). Additionally, JNK1-mediated NLRP3 phosphorylation at S194 plays a key role in NLRP3 deubiquitination (Song et al., 2017). In summary, these different intracellular signaling molecules are involved in NLRP3 inflammasome priming at both the transcriptional and post-translational levels.

\subsection{Activating of NLRP3 Inflammasome}

After the priming signal is completed, the activation signal mainly promotes the assembly and activation of NLRP3 inflammasomes and is mediated by related PAMPs or by DAMPs. NLRP3 is known to be activated by a wide variety of stimuli, including ATP, monosodium urate (MSU) crystals, nigericin, pathogenassociated RNA (Mariathasan et al., 2006; Martinon et al., 2006; Halle et al., 2008; Mao et al., 2013). Moreover, a series of molecular and cellular events, including ionic flux, overproduction of reactive oxygen species (ROS), mitochondrial dysfunction, and lysosomal damage have been demonstrated to trigger NLRP3 (Kim et al., 2021). There are certain associations between these molecular and cellular events, 


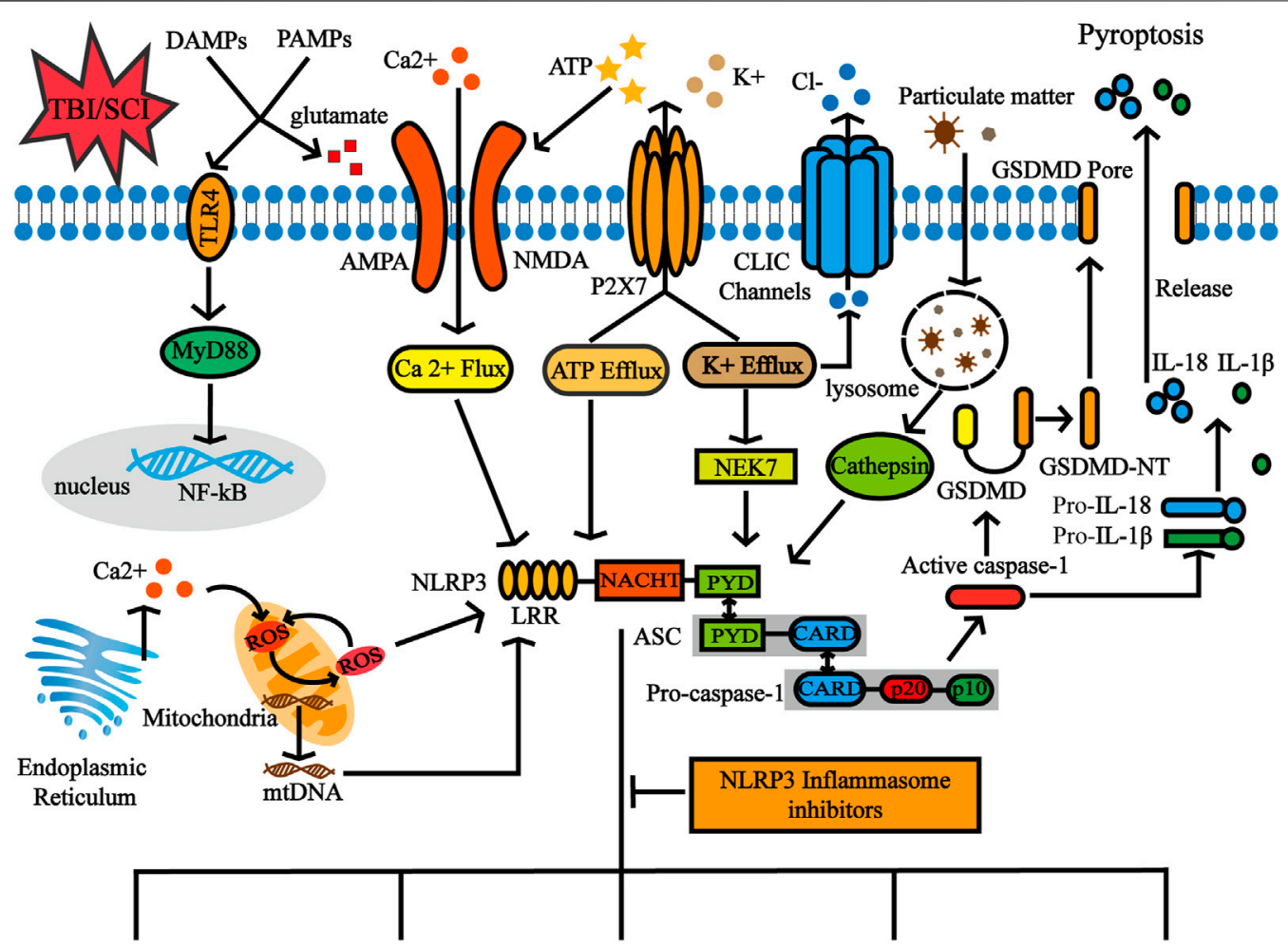

Inhibiting the polarization Increasing BBB of microglial cells score and Reducing neuronal toward the promoting apoptosis

Inhibiting inflam- Reducing cerebral M1 phenotype functional recovery mation and reducing lesions and protecting the integrity of blood - brain barrier

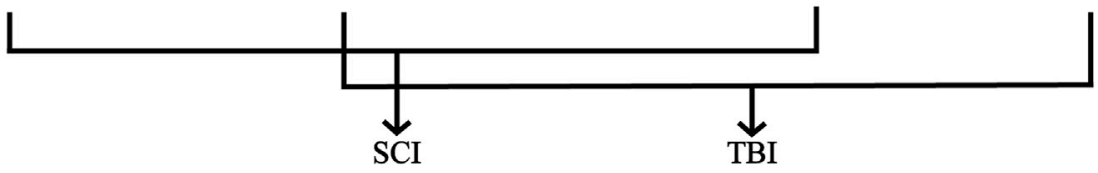

FIGURE 3 | The activation signal of NLRP3 inflammasome and the functional role of NLRP3 inflammasome inhibition in CNS post-traumatic injury. NLRP3 inflammasomes can be activated by various stimuli including ATP and particulate matter. A series of molecular and cellular events, including ionic flux, overproduction of reactive oxygen species (ROS), mitochondrial dysfunction and lysosomal damage have also been demonstrated to trigger the NLRP3. After SCI and TBI, the release of DAMPs and PAMPs is accompanied by an increase in extracellular glutamate levels, which further leads to excitotoxic neuron damage through amino 3 hydroxy- 5 methyl-4 isoxazolepropionic acid (AMPA) and N methyl-d aspartate (NMDA) receptors. Furthermore, glutamate-mediated Ca2+ influx occurs through NMDA and AMPA receptors. ATP activates the P2X7 receptor channel and releases large amounts of ATP through this channel. In addition, stimulating the P2X7 receptor results in a decrease in intracellular $\mathrm{K}+$. Inhibition of pyroptosis by NLRP3 inflammasome inhibitors affects biological processes of inflammation, proliferation, apoptosis, etc.

and although increasing studies have demonstrated their respective mechanisms, there are still some contradictions (Figure 3).

\subsubsection{Ionic Flux}

Many studies have demonstrated that changes in intracellular ion flux are the main pathways for NLRP3 inflammasome activation. Ionic flux generally includes potassium, calcium, chloride, sodium, manganese, magnesium, iron and zinc ions ( $\mathrm{Li}$ et al., 2021). $\mathrm{K}^{+}$efflux is proposed as a common ionic event for multiple-stimuli-induced NLRP3 activation (Seo et al., 2015). It was found that reduction of the intracellular $\mathrm{K}^{+}$concentration can activate the NLRP3 inflammasome signaling and mediate the maturation of IL- $1 \beta$ in response to nigericin, a $\mathrm{K}^{+} / \mathrm{H}^{+}$ionophore, and P2X purinoceptor 7 (P2X7) receptor, which binds to ATP (Perregaux and Gabel, 1994; Walev et al., 1995; Surprenant et al., 1996). P2X7 may induce the influx of cations and generate a driving force for $\mathrm{K}^{+}$efflux (Kopp et al., 2019). Importantly, the high concentration of extracellular $\mathrm{K}^{+}$hinders the activation of NLRP3 inflammasomes but does not prevent the activation of NLRC4 or AIM2 inflammasomes (Petrilli et al., 2007; MunozPlanillo et al., 2013). It is worth noting that as a necessary step for assembling the NLRP3 inflammasome, the binding of NEK7 and NLRP3 requires $\mathrm{K}^{+}$efflux (Chen et al., 2019b). This suggests that $\mathrm{K}^{+}$efflux is an upstream event of NLRP3 inflammasome activation and that $\mathrm{K}^{+}$directly regulates NLRP3. In addition, 
$\mathrm{K}^{+}$efflux may promote NLRP3 activation by inducing mitochondrial dysfunction and mitochondrial ROS (mtROS) production (Tang et al., 2017). It has recently been reported that NLRP3 inflammasome activation is independent of $\mathrm{K}^{+}$ efflux, but the specific mechanism remains unclear and needs further research in the future (Gross et al., 2016; Sanman et al., 2016; Wolf et al., 2016).

Intracellular $\mathrm{Ca}^{2+}$ mobilization is also considered to be a fundamental upstream event of NLRP3 inflammasome activation, but its role remains controversial (Murakami et al., 2012). On the one hand, Lee et al. reported that calcium-sensitive receptor (CaSR) induces $\mathrm{Ca}^{2+}$ release from endoplasmic reticulum (ER) stores, and $\mathrm{Ca}^{2+}$ promotes ASC-NLRP3 association in cell-free lysates of LPS-primed bone marrow-derived macrophage (Lee et al., 2012). Besides CaSR, intracellular $\mathrm{Ca}^{2+}$ can also be increased by ligand-gated calcium channels and voltage-dependent calcium channels (VDCCs), ER/sarcoplasmic reticulum (SR) $\mathrm{Ca}^{2+}$ stores, or the lysosome (Weber and Schilling, 2014). In addition, increased intracellular $\mathrm{Ca}^{2+}$ concentration damages mitochondria, leading to mtDNA production, mitochondrial lipid cardiolipin externalization, and mtROS release, activating NLRP3 inflammasome (Shimada et al., 2012; Iyer et al., 2013; Horng, 2014). Interestingly, this effect is mutual. The overproduction of mtROS not only leads to an increase in intracellular $\mathrm{Ca}^{2+}$ but also causes a vicious cycle of ROS, which further increases ROS and mitochondrial damage (Shimokawa et al., 2016). On the other hand, an earlier study showed that regardless of whether the cytosolic $\mathrm{Ca}^{2+}$ increases at the same time, $\mathrm{K}^{+}$ efflux can effectively activate the NLRP3 inflammasome (Katsnelson et al., 2015). Another study found that NLRP3 activation is independent of $\mathrm{Ca}^{2+}$ flux, or that NLRP3 activation is an upstream event of $\mathrm{Ca}^{2+}$ flux (Katsnelson et al., 2015).

\subsubsection{ROS and Mitochondrial Dysfunction}

Increased levels of ROS, a major metabolite of oxidative stress, trigger the activation of the NLPR3 inflammasome and the release and maturation of IL- $1 \beta$ (Vigano et al., 2015). Various sources of ROS in cells are known, and the main ones that have been deeply studied are the mitochondria and NADPH oxidase system (Mantel et al., 2012). Mitochondria play a key role in a variety of cellular functions, including ROS production, ATP production, and calcium homeostasis regulation. Mitochondrial damage can be induced by a variety of factors, such as LPS, ATP, and inhibitors of mitophagy, followed by mtROS production and mitochondrial DNA (mtDNA) release into the cytoplasm (Zhou et al., 2011; Kim et al., 2016). Mitophagy can clear damaged mitochondria and control the level of mtROS; thus, it is an important regulatory means to prevent further activation of NLRP3 (Sciarretta et al., 2012). The release of mtDNA in the cytoplasm is attributed to the opening of the mitochondrial permeability transition (MPT) pore, and the opening of the MPT pore is the result of the direct oxidation of MPT components by a large number of ROS (Nakahira et al.,
2011; Yu et al., 2019a). Shimada et al. showed that the oxidized form of mtDNA activates NLRP3 more vigorously, whereas the non-oxidized form of mtDNA is primarily inclined to activate AIM2 inflammasomes (Shimada et al., 2012).

More recently, reports also support that newly synthesized oxidative mtDNA enhances the activation of the NLRP3 inflammasome (Zhong et al., 2018). In addition, oxidation of mtDNA occurrs in severe fever with thrombocytopenia syndrome virus (SFTSV)-infected macrophages even without new mtDNA synthesis, suggesting that oxidative mtDNA is a more critical upstream event for NLRP3 inflammatory activation (Li S. et al., 2020). A recent study indicated that ELABELA (ELA) overexpression inhibits NADPH oxidase activity, which produces ROS, thereby inhibiting the activation of NLRP3 inflammasomes (Chen Z. et al., 2020). NADPH oxidase 2 (NOX2) is involved in oxidative stress in the pathological process of TBI, and the knockout of NOX2 reduces the activation of the NLRP3 inflammasome and the expression of inflammatory components (Ma et al., 2017). Moreover, NADPH oxidase four induces the activation of NLRP3 through carnitine palmitoyltransferase 1(CPT1)-mediated fatty acid oxidation (FAO) (Batista-Gonzalez et al., 2019).

\subsubsection{Lysosomal Damage}

Among several stressors that trigger the activation of NLRP3 inflammasomes, lysosomal rupture is an important factor, but the crosstalk between lysosomal rupture and NLRP3 inflammasome is not fully understood. Lysosome rupture is mainly caused by the phagocytosis of particulate matter, including silica, aluminum hydroxide, L-leucyl-L-leucine methyl ester and MSU (MunozPlanillo et al., 2013). Particulate matter in the air induces inflammation through classical or non-classical pyroptosis pathways. Previous studies have documented that airborne particulate matter (PM2.5) enters cells through endocytosis and activates NLRP3 inflammasomes to cause lung inflammation and fibrosis (Zheng R. et al., 2018). Additionally, nanoparticles produced by combustion promote the release of IL18 and IL-33 through non-classical caspase-4-dependent pathways and are not associated with NLRP3 (De Falco et al., 2017). Lysosomal rupture releases cathepsin, especially cathepsin B (CTSB), which is involved in the activation of the NLRP3 inflammasome via interaction at the endoplasmic reticulum level (Chevriaux et al., 2020).

Studies have shown that CTSB is an upstream activator of the NLRP3 inflammasome, promoting the release of IL-1 $\beta$, and this effect can be inhibited by the CTSB inhibitor CA-074-Me5 (McHugh et al., 2019; Demirel et al., 2020). Additionally, CTSB inhibits autophagy by regulating the kinase ULK1 activity, and autophagy negatively regulates the activation of NLRP3 inflammasomes (Qi et al., 2016; Zhao et al., 2021). Similarly, recent evidence has illustrated that the use of autophagy inhibitors reversed the effect of betulinic acid, which reduces the activation of pyroptosis after $\mathrm{SCI}(\mathrm{Wu}$ et al., 2021). However, evidence has shown that IL- $1 \beta$ release is not affected even in the absence of CTSB, thus casting doubt on the role of CTSB in NLRP3 inflammasome activation (Halle et al., 2008; Bauer et al., 2010; Hari et al., 2014). 


\section{DYNAMIC ACTIVATION PATTERN, AND ROLES OF NLRP3 INFLAMMASOME IN CNS TRAUMA}

Previous studies have demonstrated that NLRP3 is involved in the assembly of inflammasomes in CNS diseases and exists mainly in microglia, which are the earliest responders of CNS pathological injury (Saijo et al., 2013; Mamik and Power, 2017; Chen Y. et al., 2020). Furthermore, other researchers discovered the presence of NLRP3 inflammasome in neurons and astrocytes (Qian et al., 2017; Chen Y. et al., 2020). Since NLRP3 is the most abundant molecule among all types of inflammasomes, especially in the CNS, the dynamic activation pattern and roles of the NLRP3 inflammasome in CNS trauma was demonstrated by in vivo and in vitro experiments (Figure 4).

\subsection{NLRP3 Inflammasome in SCI}

In the early stage, after SCI, NLRP3 expression levels were remarkably upregulated in the spinal cord contusion lesion rat model at $72 \mathrm{~h}$ post-injury and in LPS + ATP-induced murine BV2 microglial cells relative to the sham model group (Liu et al., 2021). Moreover, increased protein expression of NLRP3 was also found in T13 dorsal root avulsion model rats (Ellis et al., 2016).
Consistently, in the SCI mouse model, quantitative analysis of western blot results revealed that the NLRP3 was significantly enhanced after injury. In addition to NLRP3, an immunofluorescence experiment showed an increased density of GSDMD and caspase- 1 in the spinal cord lesions, suggesting that SCI activates the NLRP3 inflammasome with the consequent increase in the pyroptosis-related markers (Wu et al., 2021).

Consistent with previous results, Zheng et al. showed that mRNA levels of NLRP3 and NLRP1 began to increase at $24 \mathrm{~h}$ in a rat model of SCI, peaked on day 3 , and gradually decreased within 7 days. Moreover, the protein expression of NLRP1 and NLRP3 was elevated within $6 \mathrm{~h}$ in primary neurons after injury. Throughout the research process, NLRP3 expression changed more significantly than NLRP1 (Zheng et al., 2019). Zendedel et al. reported that NLRP1 mRNA levels immediately rose within $6 \mathrm{~h}$ post-SCI, but this trend was completely reversed. Before 3 days, NLRP3 was always on the rise and reached its peak, whereas the NLRP1b gradually declined and reached a baseline value. After $72 \mathrm{~h}, \mathrm{NLRP} 3$ began to decline gradually until day 7 , while NLRP1b remained at its baseline level (Zendedel et al., 2016). In a recent study, they also found significant increases in ASC, NLRP3, NLRP1b, and NLRC4 mRNA expression levels after SCI in rats, as well as ASC and NLRP3 protein levels.

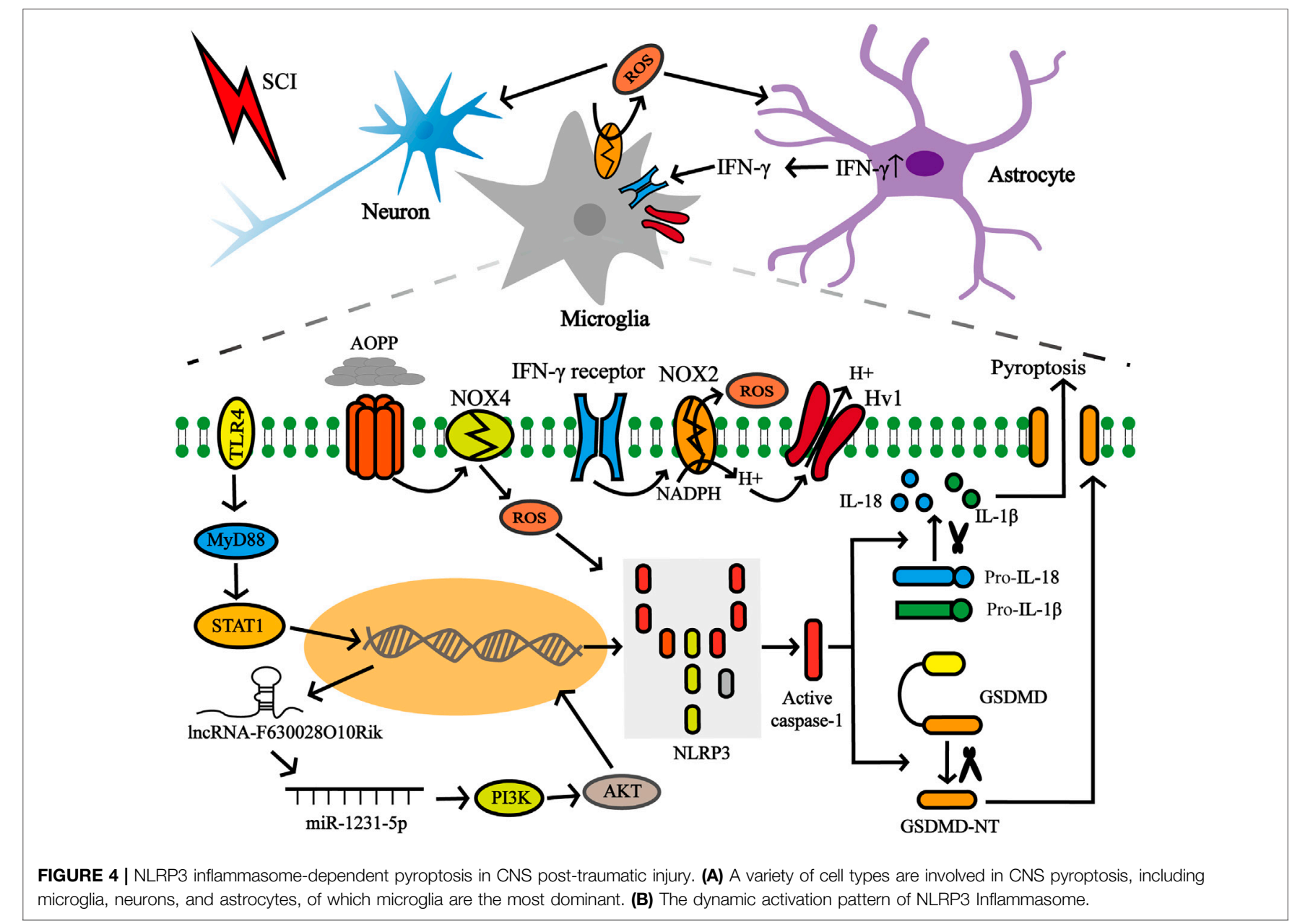


However, there was a slight decrease in the protein expression of NLRP1b, and notably, no detectable expression of NLRC4 (Zendedel et al., 2018). In addition, Jiao et al. reported that NLRP3 expression levels gradually increased after injury, peaked on the second day, and then gradually decreased in SCI mice (Jiao et al., 2020). Clinically, the authors of a study reported that the level of NLRP3 expression in the peripheral blood samples of SCI patients was significantly higher than that in normal patients and was positively related to the severity of the injury. In particular, they also found that compared with the NLRP3/GSDMD low expression group, the high level group of the NLRP3/GSDMD had a higher neck disability index (NDI) and a lower Japanese Orthopedic Association (JOA) score (Xu et al., 2021).

Additional studies have compared the dynamic expression pattern of the pyroptosis-related key gene NLRP3 after SCI (Figure 5). $\mathrm{Xu}$ et al. showed that SCI injury signals are transmitted to microglia via TLR4/MyD88, which phosphorylate STAT1 and trigger the lncRNAF630028O10Rik/miR-1231-5p pathway, and finally enhance pyroptosis via $\mathrm{PI} 3 \mathrm{~K} / \mathrm{AKT}$. In the injured spinal cord segment of TLR4 -/-mice, the protein and mRNA expression levels of NLRP3 were sharply reduced 3 days post-injury, as well as in BV2 microglial cells treated with the TLR4 inhibitor TAK242 compared to the wild-type (WT) sham-operated group (Xu et al., 2020). As mentioned earlier, the increase in ROS levels is a common event that activates NLRP3 inflammasomes and is regulated by the voltage-gated proton channel (Hv1) (Patel et al., 2019). The latest research shows that Hv1 in spinal microglia increases significantly after spinal nerve transection and contributes to the production of ROS. ROS act on neurons and astrocytes, causing IFN- $\gamma$ release, which in turn further activates microglia and microglia-astrocyte interactions (Peng et al., 2021). The number of NLRP3-positive neurons in SCI mice lacking Hv1 was always significantly lower than that in the WT group, and reached a peak on the third day after the injury, and the number on the seventh day was lower than that on the first day (Li et al., 2020c). Additionally, NADPH oxidase-mediated ROS production was closely associated with advanced oxidation protein products (AOPPs) and presented in a time- and dosedependent manner (Sun et al., 2016). Liu et al. found that AOPP levels in the plasma, cerebrospinal fluid and spinal cord were

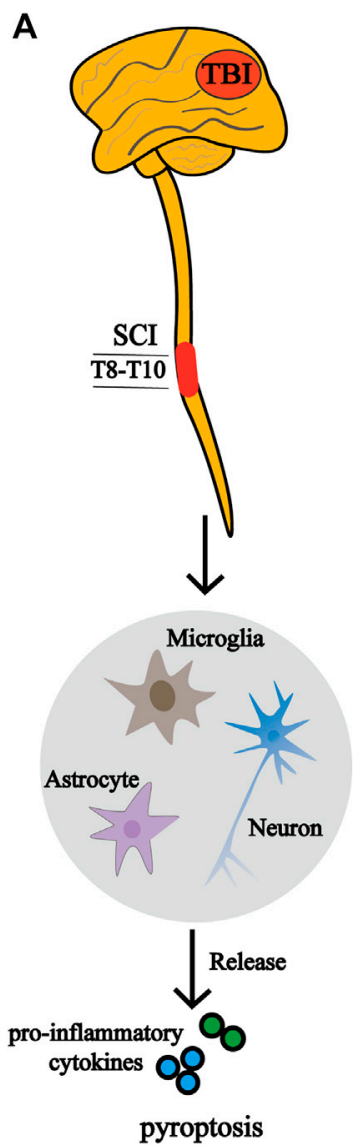

B
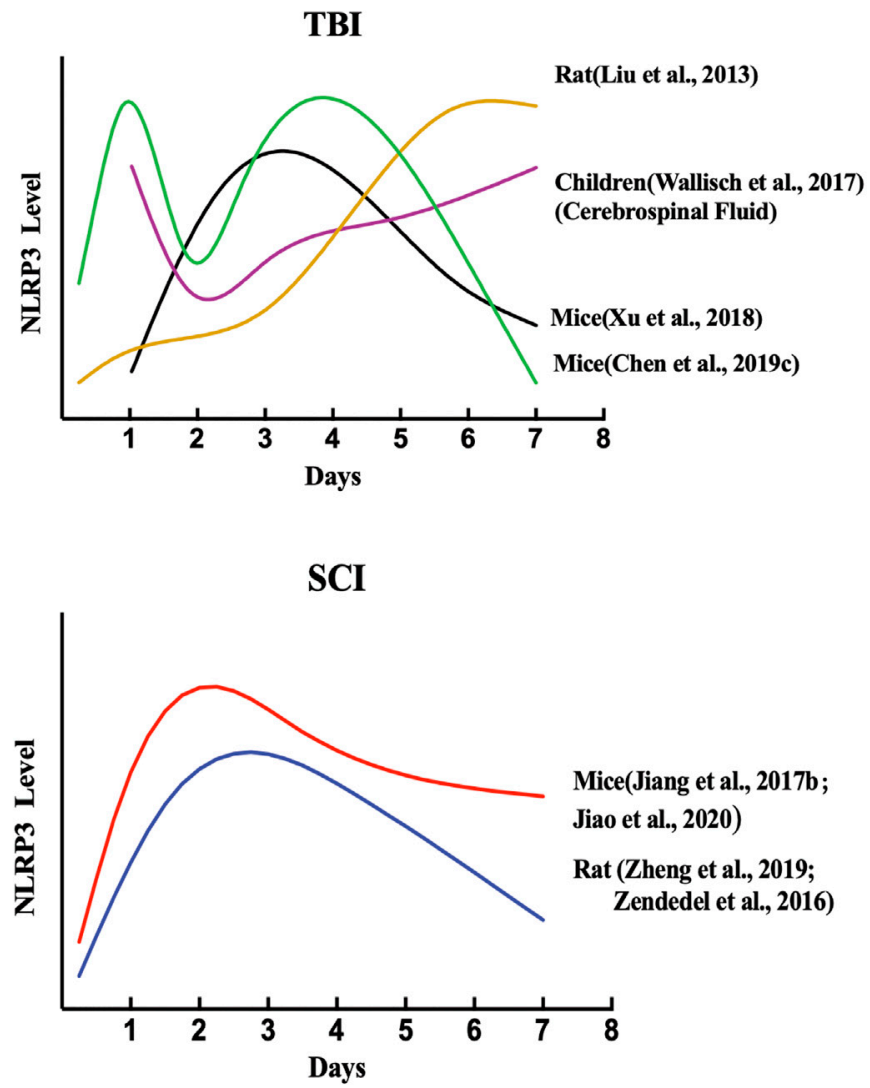

FIGURE 5 | Factors affecting NLRP3 in microglia after SCI. SCl injury signals were transmitted to microglia via TLR4/MyD88/STAT1 network, which subsequently activated the IncRNA-F630028010Rik/miR-1231-5p pathway, and subsequently triggered pyroptosis. In addition, the increase of Hv1 in microglia post-injury contributes to ROS production. ROS production promotes IFN- $\gamma$ release from astrocytes, and IFN- $\gamma$ in turn strengthens microglia activation and microglia-astrocyte interaction. ROS production is also closely related to AOPP. 
elevated after SCI and peaked on day 3, followed by a gradual decline, similar to the trend detected in NLRP3 after SCI. The authors have also demonstrated that compared to BV2 cells incubated with LPS + ATP, the relative band ratio of NLRP3/ GAPDH was slightly higher in the AOPPS-MSA group $(50 \mu \mathrm{g} /$ $\mathrm{ml})$, but remarkably enhanced in the AOPPS-MSA group (100 and $200 \mu \mathrm{g} / \mathrm{ml}$ ) (Liu Z. et al., 2020).

\subsection{NLRP3 Inflammasome in TBI}

TBI also induces the expression of NLRP3 inflammasome components (NLRP3, ASC, and caspase-1) in the cerebral cortex, cerebrospinal fluid (CSF), and peripheral blood in both humans and rodents. In the peri-contusional cortex $24 \mathrm{~h}$ after injury, the protein and mRNA levels of NLRP3, ASC, and caspase-1 were augmented mainly in mice with cold brain injury (Wei et al., 2016). In addition, controlled cortical impact (CCI) induced significant expression of NLRP1, NLRP3, NLRC4, ACS, and caspase-1 in brain microvascular endothelial cells from the injured cerebral cortex of mice at 3 days (Ge et al., 2018). High expression of NLRP3 and caspase-1 was also detected in blast-induced TBI rat models at 12 and $24 \mathrm{~h}$ post-injury, and rats were subjected to penetrating ballistic-like brain injury after $4 \mathrm{~h}$ (Ma et al., 2016; Lee et al., 2018). More importantly, NLRP3 elevated levels were examined in the peripheral blood of neonatal hypoxic-ischemic encephalopathy patients and the CSF of children with severe TBI(Wallisch et al., 2017; Lv et al., 2020). Histologically, NLRP3-/- mice subjected to an impact-acceleration model of diffuse TBI showed less damage to brain structures compared to WT mice (Irrera et al., 2017). ASC levels in serum and serumderived extracellular vesicles were markedly higher in TBI patients with lung injury than in patients without complications (Kerr et al., 2019). ASC neutralization markedly reduced caspase- 1 activation and cerebral contusion volume after post-TBI (de Rivero Vaccari et al., 2009).

Liu et al. reported that NLRP3 mRNA expression began to increase and reached a peak value at $6 \mathrm{~h}$ post-TBI in rats using the modified Feeney's model. After this peak, NLRP3 expression gradually decreased within $24 \mathrm{~h}$, but then continued to increase until 7 days, and its expression was higher than that at $6 \mathrm{~h}$. Notably, in the same study, NLRP3 protein levels continued to increase significantly over time after injury (Liu et al., 2013). Similarly, CSF concentration of NLRP3 in children with severe TBI instantly rose and peaked at $24 \mathrm{~h}$, then it declined at day 2 but was still higher than that in the control group, and then increased again at day 3. In addition, they also found that young TBI children with TBI $<5$ years old have higher levels of NLRP 3 in the CSF than patients $\geq 5$ years old, indicating a worse poor outcome (Wallisch et al., 2017). A possible explanation for this similar trend is that after TBI, disturbances in cellular ionic homeostasis, including $\mathrm{K}^{+}$efflux and $\mathrm{Ca}^{2+}$ influx, and the outflow of ATP from dying cells and traumatic lysis led to the first elevation of NLRP3. The latter increase in NLRP3 may be due to secondary injury, including cellular stress and inflammatory cascade (Liu et al., 2013). Interestingly, unlike previous studies, authors from a study documented that, in the TBI mouse model, NLRP3 mRNA levels gradually increased and peaked at $24 \mathrm{~h}$, then fell gradually until the next day, rose again within $72 \mathrm{~h}$, and finally gradually decreased at day 7 (Chen et al., 2019c). In the peri-contusional cortex, the protein levels of NLRP3, caspase-1, and ASC started to increase at day 1 and peaked at $72 \mathrm{~h}$ in mice were subjected to the CCI injury; on day 7 , the protein expression was decreased but remained higher than in controls ( $\mathrm{Xu}$ et al., 2018). Therefore, this suggests that the dynamic expression pattern of NLRP3 is influenced by multiple factors. Moverover, understanding the time-course change of the NLRP3 inflammasome contributed to the determination of the appropriate dose, dose interval, and frequency of drug administration, which may improve the therapeutic effect.

Data from several experiments also suggest that not all inflammasome components increase after injury. Immunoblot analysis results demonstrated that caspase-1, caspase-11, and ASC substantially increased in the rat model 15 min following a moderate parasagittal fluid-percussion injury, but NLRP1 did not change significantly (de Rivero Vaccari et al., 2009). In addition, Liu et al. showed that NLRP3 mRNA levels changed significantly after TBI, except for pro-caspase1 (Chen et al., 2019c). In children with severe TBI, NLRP1 in the CSF was detected in only a small number of patients and was elevated only during the first day and undetectable thereafter (Wallisch et al., 2017). With the activation of NLRP3, highly expressed IL-1 $\beta$ and IL-18 were detected in the CSF of TBI patients and the serum of SCI patients and these cytokines are closely related to the pathogenesis of neuroinflammation (Helmy et al., 2011; Kerr et al., 2018; Jiao et al., 2020). Interestingly, the dynamic expression patterns of not only NLRP3 inflammatory components after CNS injury but also inflammatory cytokines, vary widely in the literature. In most cases, the time course of IL$1 \beta$ and IL-18 was consistent with the changes in NLRP3, suggesting that NLRP3 inflammasome activation is involved in the inflammatory response after CNS injury.

\section{NLRP3 INFLAMMASOME INHIBITORS IN CNS TRAUMA}

The NLRP3 inflammasome, as a central part of the cell pyroptosis process, is extensively involved in CNS injury, making it an attractive therapeutic target. Various promising NLRP3 inhibitors have been reported, although none have been approved for the treatment of SCI and TBI. In this paper, several important NLRP3 inhibitors and their mechanisms have been described. (Table 1).

\subsection{MCC950}

MCC950, a diarylsulfonylurea-containing compound, is considered to inhibit the processing of IL-1 $\beta$ and is also known as a highly potent inhibitor of NLRP3 (Perregaux et al., 2001). Experiments have been conducted in recent years to explore the potential therapeutic effects of MCC950 and have been demonstrated in several disease models, such as diabetes and Alzheimer's disease (Dempsey et al., 2017; Ward et al., 2019). In addition, MCC 950 inhibited caspase- 1 activation and subsequent genotype-related IL- $1 \beta$ increase in a murine model of TBI $(\mathrm{Xu}$ 
TABLE 1 | Specific inhibitory compounds of NLRP3 inflammasome activation.

\begin{tabular}{|c|c|c|c|c|c|}
\hline Agents & Host & $\begin{array}{l}\text { Animal } \\
\text { model }\end{array}$ & $\begin{array}{l}\text { Pharmacological } \\
\text { effects }\end{array}$ & Specificity & References \\
\hline \multirow[t]{2}{*}{ MCC950 } & $\begin{array}{l}\text { Primary cortical } \\
\text { neurons }\end{array}$ & $\mathrm{SCl}$ & $\begin{array}{l}\text { Reducing neuronal apoptosis and promoting functional } \\
\text { recovery }\end{array}$ & NLRP3 & Ismael et al. (2018), He et al. (2020a) \\
\hline & C57BL/6 mice & $\mathrm{TBI}$ & $\begin{array}{l}\text { Reducing neurological severity score and brain water content, } \\
\text { repressing NLRP3, ASC, caspase-1, IL-1 } 3 \text {, TNF-a, NF-kB/p65, } \\
\text { caspase-3, and PARP after TBI }\end{array}$ & & \\
\hline CY-09 & SD rats & $\mathrm{SCl}$ & $\begin{array}{l}\text { NACHT ATPase inhibitor; binding to Walker A motif; and } \\
\text { inhibiting BzATP }\end{array}$ & NLRP3 & Fan et al. (2020) \\
\hline \multirow[t]{2}{*}{$\begin{array}{l}\text { Bay 11- } \\
7082\end{array}$} & C57BL/6 mice & $\mathrm{SCl}$ & $\begin{array}{l}\text { Reducing neuronal death promoting motor recovery and } \\
\text { alleviating secondary injury after } \mathrm{SCl}\end{array}$ & NLRP3 & $\begin{array}{l}\text { Jayakumar et al. (2014), Jiang et al. (2017b), } \\
\text { Zheng et al. (2018a), Jiang et al. (2019) }\end{array}$ \\
\hline & $\mathrm{CCl}$ rats & $\mathrm{TBI}$ & $\begin{array}{l}\text { Reducing increased brain water content in rats after fluid } \\
\text { percussion injury and alleviating TBI-induced injury }\end{array}$ & NLRC4 & \\
\hline \multirow[t]{2}{*}{ Tranilast } & SD rats & $\mathrm{SCl}$ & $\begin{array}{l}\text { Inhibiting SCl acute stage inflammation and fibrosis, confining } \\
\text { lesions around the cavity }\end{array}$ & NLRP3 & Hanada et al. (2014), Lu et al. (2020) \\
\hline & KunMing mice & $\mathrm{TBI}$ & $\begin{array}{l}\text { Down-regulating phosphodiesterase-4 B (PDE4B) expression, } \\
\text { inhibiting neuroinflammation and reducing bleeding areas in TBI } \\
\text { mice }\end{array}$ & & \\
\hline Rubesin & C57BL/6 mice & $\mathrm{TBI}$ & $\begin{array}{l}\text { Inhibiting NLRP3 inflammasome and subsequent secretion of } \\
\text { IL1 } \beta \text { and IL-18, preventing neuronal apoptosis, reducing } \\
\text { cerebral edema, and protecting the integrity of BBB }\end{array}$ & NLRP3 & Yan et al. (2020) \\
\hline MiR-223 & $\mathrm{CCl}$ mice & $\mathrm{SCl}$ & $\begin{array}{l}\text { Reducing the neuropathic pain caused by } \mathrm{CCl} \text {, decreasing the } \\
\text { expression of apoptosis and inflammatory factors, and } \\
\text { increasing the proportion of } \mathrm{M} 2 \text { macrophages }\end{array}$ & NLRP3 & Zhang et al. (2020), Zhu et al. (2021) \\
\hline $\begin{array}{l}\text { MiR- } \\
423-5 p\end{array}$ & $\mathrm{SD}$ rats & $\mathrm{SCl}$ & $\begin{array}{l}\text { Inhibiting the polarization of microglial cells toward the M1 } \\
\text { phenotype }\end{array}$ & NLRP3 & Cheng et al. (2021) \\
\hline MiR-34c & C57BL/6 mice & $\mathrm{SCl}$ & $\begin{array}{l}\text { Attenuating } \mathrm{CCl} \text {-induced neuropathic pain and decreasing } \\
\text { inflammatory factors }\end{array}$ & NLRP3 & Xu et al. (2019) \\
\hline MiR-193a & C57BL/6 mice & $\mathrm{TBI}$ & $\begin{array}{l}\text { Suppression of miR-193a significantly reduced post-traumatic } \\
\text { neuroinflammation and improved neurofunctional recovery }\end{array}$ & NLRP3 & Si et al. (2020) \\
\hline
\end{tabular}

et al., 2018). A recent study by Baker et al. demonstrated that MCC950 can reverse caspase-4/5-induced IL-1 $\beta$ production and cell death in cells transfected with LPS (Baker et al., 2015). Therefore, MCC950 specifically targets the NLRP3 inflammasome and inhibits the activation of the canonical and non-canonical NLRP3 inflammasome, thus blocking pyroptotic cell death and IL-1 $\beta$ release in response to NLRP3 stimuli. Correspondingly, Coll et al. discovered that although MCC950 blocked NLRP3-induced ASC oligomerization, it did not suppress the priming phase of NLRP3 activation, as well as NLRC4 and AIM2. They further found that MCC950 also hindered IL- $1 \beta$ secretion but did not affect TNF- $\alpha$ in a dosedependent manner. More specifically, the inhibitory effect of MCC950 may target upstream signaling pathways of the NLRP3 inflammasome because it does not directly inhibit NLRP3-ASC, NEK7-NLRP3, or NLRP3-NLRP3 interactions (Coll et al., 2015). In a recent study, MCC950 was shown to dose-dependently block nigerinin-induced chloride efflux, which may be evidence that MCC950 inhibits upstream signaling events of NLRP3 activation (Jiang H. et al., 2017). However, other upstream ion signaling events, such as $\mathrm{K}^{+}$efflux and $\mathrm{Ca}^{2+}$ flux, were not blocked by MCC950 (Coll et al., 2015). Ismael et al. reported that MCC950 significantly inhibited the cleavage of caspase- 3 and poly (ADPribose) polymerase (PARP), a proapoptotic marker. In addition, the reduction of caspase- 3 was associated with the repression of MCC950-associated NF-kB/p65, which strongly indicated the protective effect of MCC950 on apoptotic cell death in TBI lesions (Ismael et al., 2018). Importantly, Xu et al. found that the neuroprotective effect of MCC950 and its ability to reduce cerebral edema were dependent on the presence of microglia and that the therapeutic window of MCC950 was limited within $6 \mathrm{~h}$ post-TBI(Xu et al., 2018). Compared with a single drug treatment, MCC950 combined with rapamycin-induced mitophagy further blocked NLRP3 inflammasome assembly and enhanced the neuroprotection in CCI mice (Chen et al., 2019c). Furthermore, MCC950 significantly reduced neuronal apoptosis, improved neuronal survival and functional recovery, and reduced scar formation in SCI mice (He et al., 2020a; Jiao et al., 2020). Although it will take some time for MCC950 to be switched to clinical agents, it represents an important strategy for targeting or addressing CNS trauma because of its specificity and effectiveness in NLRP3 inhibition, as well as its long-term safety and neuroprotective effects of oral administration (Gordon et al., 2018).

\subsection{CY-09}

There is evidence that the ATPase activity of the NACHTdomain of NLRP3 is critical for NLRP3 oligomerization (Duncan et al., 2007). The ATPase activity of NLRP3 has also received extensive attention as a drug target, with a large number of compounds currently receiving attention. Jiang et al. found that a small-molecule CY-09 competitively binds to the Walker A motif of NLRP3, but not Walker B, although the Walker B motif is also necessary for ATPase activity, thus eliminating NLRP3 
binding to ATP and blocking its ATPase activity. Notably, CY-09 inhibition also specifically targets NLRP3 ATPase activity and does not affect purified NLRP1, NLRC4, NOD2, or RIG-I ATPase activity. Similarly, CY-09 did not affect LPS-induced priming and TNF- $\alpha$ production (Jiang H. et al., 2017). Unlike MCC950, CY-09 did not block nigerin-induced chloride efflux but effectively inhibited the production of IL- $1 \beta$ and neutrophil influx induced by MSU injection (Jiang H. et al., 2017). Current studies have shown that CY-09 dose-dependently inhibits caspase- 1 activation and IL- $1 \beta$ production, but the inhibition of non-classical NLRP3 inflammasome activation requires further study. In microglia of SCI rats, CY-09 significantly inhibited 3'-O-(4-benzoylbenzoyl) adenosine $5^{\prime}$-triphosphate (BzATP), which regulates the NLRP3 inflammasome by upregulating the P2X7 receptor, a key inflammation switch, and ultimately inhibits the development of neuroinflammation (Fan et al., 2020). Increased GFAP expression was significantly attenuated, and astrocyte activation was inhibited by pretreatment with CY-09, which contributes to the prognosis of SCI and TBI (Shen et al., 2020). CY-09 exhibits good pharmacokinetics and directly targets NLRP3 in vivo, thus providing a novel strategy to inhibit NLRP3 inflammasome activation.

\subsection{Bay 11-7082}

BAY 11-7082 is a sulfonic derivative with a variety of pharmacological activities. Similar to CY-09, Juliana et al. reported that BAY 11-7082 blocked NLRP3-induced ASC formation by inhibiting NLRP3 ATPase activity, and its effect was dependent on the vinyl sulfone group. However, Juliana et al. observed that Bay 11-7082 did not inhibit NLRP1 ATPase activity, but could partially inhibit NLRC4 inflammasome activation, although the exact mechanism is still unclear (Juliana et al., 2010). Moreover, BAY 11-7082 inhibited microglia/macrophage activation and neutrophil infiltration, reduced glial proliferation and improved mitochondrial dysfunction, thereby reducing neuronal death and promoting motor recovery in SCI mice (Jiang W. et al., 2017). Notably, BAY 11-7082 contributed to improving secondary lung injury after SCI (Jiang et al., 2019). Additionally, in rats subjected to the CCI injury, Zheng et al. showed that the synergistic effect of the coadministration of Bay-11-7082 and dexmedetomidine improved neuronal activity and inhibited microglial activation, as well as hippocampus tissues inflammation (Zheng B. et al., 2018). BAY 11-7082 is also an NF- $\mathrm{BB}$ inhibitor that irreversibly represses TNF- $\alpha$-induced phosphorylation of I $\mathrm{IK}-\alpha$ and subsequent nuclear translocation of NF- $\mathrm{kB}$ (Jing et al., 2012). In rats inoculated with breast carcinoma cell Walker-256, Wang et al. found a significant increase in monocyte chemoattractant protein-1 (MCP-1)/chemokine CC motif receptor-2 (CCR2) and NF- $\kappa \mathrm{B}$ expression in spinal cord neurons, but this effect was inhibited by treatment with BAY 11-7082 (Wang et al., 2018).

A report from Jayakumar showed that BAY 11-7082 administration significantly inhibited NF- $\mathrm{KB}$ activation and swelling of astrocytes, inhibited the viability of $\mathrm{Na}^{+}, \mathrm{K}^{+}, 2 \mathrm{Cl}^{-}$ cotransporter, and reduced the high brain water content in rats after fluid percussion injury (Jayakumar et al., 2014).
Interestingly, BAY 11-7082 administration inhibited Snail expression increase, the main inducer of epithelialmesenchymal transition (Jing et al., 2012). Ripk1 is an important upstream regulator of necroptosis and is significantly upregulated after moderate TBI(Zhang et al, 2017). Ripk1 overexpression promoted the activation of the NF- $\mathrm{KB}$ signaling pathway and contributed to the release of pro-inflammatory factors and the inhibition of autophagyrelated proteins. BAY 11-7082 treatment significantly eliminated these effects and alleviated the TBI-induced injury (Liu J. et al., 2020). Tests on Bay11-7082 are still mostly limited to animals, although they show limited toxicity (Keller et al., 2006). According to the published literature, Bay11-7082 has shown good therapeutic effects in CNS trauma, and future work needs to transition to clinical trials to test its safety and therapeutic effect.

\subsection{Tranilast}

Tranilast is an analog of the tryptophan metabolite and has been used to treat bronchial asthma since 1982 because of its potent anti-inflammatory properties (Darakhshan and Pour, 2015). A recent report by Huang et al. clarified the mechanism underlying the anti-inflammatory effect of tranilast. Like CY-09 and Bay 117082, tranilast is also an inhibitor that targets NLRP3. However, tranilast binds directly to the NACHT domain of NLRP3, inhibiting the interaction and oligomerization of NLRP3NLRP3 and NLRP3 -ASC, but not NLRP3 -NEK7, thereby blocking the assembly of the NLRP3 inflammasome. In addition, tranilast did not affect NLRP3 ATPase activity and specifically inhibited the NLRP3 inflammasome but did not inhibit other inflammasome (Huang et al., 2018). Futhermore, Hanada et al. found that tranilast inhibited inflammation in the acute phase of SCI and confined lesions around the cavity, reducing the formation of TGF- $\beta$-mediated fibrotic scarring. They noted that tranilast is safer to administer orally compared to the risk of embolism associated with intravenous administration (Hanada et al., 2014). Second, the combined delivery of tranilast and methylprednisolone had a greater therapeutic effect than the single delivery of either (Mbori et al., 2016). Compound 5, a novel rolipram-tranilast hybrid, has been shown to have strong neuroprotective potential, significantly inhibiting neuroinflammation, and markedly reducing bleeding areas in TBI mice (Lu et al., 2020). Tranilast has been clinically used in other diseases to demonstrate its safety and efficacy. In vivo experiments have demonstrated significant therapeutic and prophylactic effects in SCI and TBI mouse models. To strengthen clinical relevance, further studies are needed to identify the tranilast oral dose, treatment window, and more detailed therapeutic effects and mechanisms.

\subsection{Rubesin}

Rubesin is a natural, biologically active diterpenoid derived from the herb Rabdosia, which has been found to have antiinflammatory, antioxidant and anti-tumor properties (Yu et al., 2019b). Similar to the specific inhibitors mentioned above, oridonin does not interfere with the upstream signaling pathway of the NLRP3 inflammasome and acts directly on the NLRP3 inflammasome itself. In contrast to tranilast, oridonin 
blocks NLRP3 inflammasome assembly by blocking the NLRP3NEK7 interaction, but does not affect NLRP3-NLRP3 and NLRP3-ASC. Mechanistically, oridonin specifically binds to the NACHT domain of NLRP3 and forms covalent bonds with cysteine 279 in the NACHT domain, thereby blocking the interaction between NLRP3 and NEK7. In this study, we also found that oridonin did not affect the activation of AIM2 and NLRC4 inflammasomes and the production of TNF- $\alpha$ (He et al., 2018). Activation of the NLRP3 inflammasome and subsequent secretion of pro-inflammatory cytokines IL-1 $\beta$ and IL-18 were significantly inhibited in ORidonin-treated TBI mice. Moreover, oridonin effectively prevented neuronal apoptosis, reduced cerebral edema, and protected the integrity of $\mathrm{BBB}$, showing a strong neuroprotective function (Yan et al., 2020). Although oridonin has shown good therapeutic effects in rheumatoid arthritis, colitis and other inflammatory diseases, it has been shown to be able to treat NLRP3-driven diseases (Liu et al., 2016; He S.-D. et al., 2020). However, there is still a large gap in the study of neuroinflammation in CNS trauma, which needs to be addressed by further research.

\subsection{MIR-223}

MicroRNAs (miRNAs) are small and endogenous non-coding RNA molecules that down-regulate gene expression at the posttranscriptional level (Miyake et al., 2012). MiR-223 is one of the microRNAs that is known to be associated with inflammation and has attracted increasing attention. Bauernfeind et al. revealed that the conserved sites in the 3'untranslated regions of the NLRP3 transcript were bound to the myeloid-specific microRNA miR-223, thus dampening NLRP3 expression and diminishing IL- $1 \beta$ secretion. Meanwhile, miR-223 antagonists contribute to increased levels of NLRP3 protein. Importantly, miR-223 did not affect activation of the AIM2 inflammasome (Bauernfeind et al., 2012). A previous study revealed that MiR223 can directly inhibit NLRP3 expression, reduce cerebral edema, and inhibit the secretion of inflammatory factors after intracerebral hemorrhage (Yang et al., 2015). Moreover, miR-223 not only protects the brain against glutamate excitotoxicity but also protects dissociated cortical neurons from degeneration in peripheral blood mononuclear cell-conditioned media (Harraz et al., 2012; Morquette et al., 2019). Studies have shown that miR223 levels are significantly increased after acute SCI, and inhibition of miR-223 aggravates injury and inflammatory response, while overexpression has the opposite effect. The author and colleagues also found that overexpression of miR223 in microglia inhibits LPS-induced inflammation (Zhang et al., 2020). In addition to what has been described above, several other miRNAs have been shown to be involved in the activation of NLRP3 inflammasome and have shown efficacy in CNS trauma disease models, including miR-423-5p, miR-34c, and miR-193a (Xu et al., 2019; Si et al., 2020; Cheng et al., 2021). Interestingly, downregulation of circ 0001723 induces NLRP3 expression by upregulating miR-380-3p (Li et al., 2020b). In addition, LncRNA-Meg3 negatively regulates miR-7a-5p to induce microglial inflammation by regulating NLRP3 (Meng et al., 2021). Thus, it is also possible to target miRNAs by regulating other types of non-coding RNAs, such as circRNAs and lncRNAs, thereby regulating NLRP3.

\section{CONCLUSION}

The pathophysiological processes in SCI and TBI are complicated, and the identification of novel therapeutic targets is urgently needed. Activation of the NLRP3 inflammasome is a central link in pyroptosis and is involved in the pathological development of various inflammatory diseases, including SCI and TBI. While our understanding of the mechanisms of NLRP3 inflammasome priming, activation, and post-translational modification is improving, therapies targeting NLRP3 are advancing rapidly. However, the current treatment of NLRP3 pathology is still limited to animal experiments and is even more restricted to the canonical pyroptosis pathway. It is also important for other NLR or inflammasome sensors or caspases in the non-canonical pyroptosis pathway. The NLRP3 dynamic expression pattern in SCI and TBI shows that the NLRP3 time course of different models has a similar trend. Drug administration can be determined using the corresponding dose and absorption efficiency at different time points in a certain model, which may improve the therapeutic effect. Additionally, it can be combined with clinically effective drugs, such as methylprednisolone. We can also focus on the role of non-coding RNAs in pyroptosis in TBI and SCI. Recent research has shown that bone marrow mesenchymal stem cell-derived exosomes significantly inhibit pyroptosis to improve SCI, and the potential mechanism is to regulate NOD1-related signaling pathways (Zhou et al., 2022). Despite the increasing exosomes from different sources or those carrying a variety of non-coding RNAs, few studies directly target NLRP3 and there are still large gaps in CNS trauma. In summary, further work is still needed to elucidate the role of NLRP3 inflammasome in CNS injury, but increasing drugs are being developed and novel therapies are quickly becoming a reality.

\section{AUTHOR CONTRIBUTIONS}

$\mathrm{CZ}$ and JZ collected the information and drafted and revised the manuscript. YF and JZ contributed to collecting information and editing of the manuscript. JW directed the work and finalized the manuscript. All authors have read and approved the final manuscript.

\section{FUNDING}

This project was supported by the National Natural Science Foundation of China (Grant Nos 81372046 and 81571928). 


\section{REFERENCES}

Allam, R., Lawlor, K. E., Yu, E. C. W., Mildenhall, A. L., Moujalled, D. M., Lewis, R. S., et al. (2014). Mitochondrial Apoptosis Is Dispensable for NLRP 3 Inflammasome Activation but Non-apoptotic Caspase-8 Is Required for Inflammasome Priming. EMBO Rep. 15 (9), 982-990. doi:10.15252/ embr.201438463

Baker, P. J., Boucher, D., Bierschenk, D., Tebartz, C., Whitney, P. G., D'Silva, D. B., et al. (2015). NLRP3 Inflammasome Activation Downstream of Cytoplasmic LPS Recognition by Both Caspase-4 and Caspase-5. Eur. J. Immunol. 45 (10), 2918-2926. doi:10.1002/eji.201545655

Batista-Gonzalez, A., Vidal, R., Criollo, A., and Carreño, L. J. (2019). New Insights on the Role of Lipid Metabolism in the Metabolic Reprogramming of Macrophages. Front. Immunol. 10, 2993. doi:10.3389/fimmu.2019.02993

Bauer, C., Duewell, P., Mayer, C., Lehr, H. A., Fitzgerald, K. A., Dauer, M., et al. (2010). Colitis Induced in Mice with Dextran Sulfate Sodium (DSS) Is Mediated by the NLRP3 Inflammasome. Gut 59 (9), 1192-1199. doi:10.1136/ gut.2009.197822

Bauernfeind, F. G., Horvath, G., Stutz, A., Alnemri, E. S., MacDonald, K., Speert, D., et al. (2009). Cutting Edge: NF-Kb Activating Pattern Recognition and Cytokine Receptors License NLRP3 Inflammasome Activation by Regulating NLRP3 Expression. J. Immunol. 183 (2), 787-791. doi:10.4049/ jimmunol.0901363

Bauernfeind, F., Rieger, A., Schildberg, F. A., Knolle, P. A., Schmid-Burgk, J. L., and Hornung, V. (2012). NLRP3 Inflammasome Activity Is Negatively Controlled by miR-223. J. Immunol. 189 (8), 4175-4181. doi:10.4049/jimmunol.1201516

Boon, H., Kostovski, E., Pirkmajer, S., Song, M., Lubarski, I., Iversen, P. O., et al. (2012). Influence of Chronic and Acute Spinal Cord Injury on Skeletal Muscle $\mathrm{Na}+-\mathrm{K}+-\mathrm{ATPase}$ and Phospholemman Expression in Humans. Am. J. Physiology-Endocrinology Metab. 302 (7), E864-E871. doi:10.1152/ ajpendo.00625.2011

Boucher, D., Monteleone, M., Coll, R. C., Chen, K. W., Ross, C. M., Teo, J. L., et al. (2018). Caspase-1 Self-Cleavage Is an Intrinsic Mechanism to Terminate Inflammasome Activity. J. Exp. Med. 215 (3), 827-840. doi:10.1084/ jem.20172222

Breitbach, K., Sun, G. W., Köhler, J., Eske, K., Wongprompitak, P., Tan, G., et al. (2009). Caspase-1 Mediates Resistance in Murine Melioidosis. Infect. Immun. 77 (4), 1589-1595. doi:10.1128/IAI.01257-08

Bryukhovetskiy, A. S. (2018). Translational Experience of 28 Years of Use of the Technologies of Regenerative Medicine to Treat Complex Consequences of the Brain and Spinal Cord Trauma: Results, Problems and Conclusions. J. Neurorestoratology 1 (1), 16-31. doi:10.26599/jnr.2018.9040009

Chen, X., Liu, G., Yuan, Y., Wu, G., Wang, S., and Yuan, L. (2019a). NEK7 Interacts with NLRP3 to Modulate the Pyroptosis in Inflammatory Bowel Disease via NF-Kb Signaling. Cell Death Dis 10 (12), 906. doi:10.1038/s41419-019-2157-1

Chen, Y., Li, Y., Guo, L., Hong, J., Zhao, W., Hu, X., et al. (2020a). Bibliometric Analysis of the Inflammasome and Pyroptosis in Brain. Front. Pharmacol. 11, 626502. doi:10.3389/fphar.2020.626502

Chen, Y., Meng, J., Bi, F., Li, H., Chang, C., Ji, C., et al. (2019b). NEK7 Regulates NLRP3 Inflammasome Activation and Neuroinflammation Post-traumatic Brain Injury. Front. Mol. Neurosci. 12, 202. doi:10.3389/fnmol.2019.00202

Chen, Y., Meng, J., Xu, Q., Long, T., Bi, F., Chang, C., et al. (2019c). Rapamycin Improves the Neuroprotection Effect of Inhibition of NLRP3 Inflammasome Activation after TBI. Brain Res. 1710, 163-172. doi:10.1016/ j.brainres.2019.01.005

Chen, Z., Wu, C., Liu, Y., Li, H., Zhu, Y., Huang, C., et al. (2020b). ElABELA Attenuates Deoxycorticosterone Acetate/salt-Induced Hypertension and Renal Injury by Inhibition of NADPH oxidase/ROS/NLRP3 Inflammasome Pathway. Cel Death Dis 11 (8), 698. doi:10.1038/s41419-020-02912-0

Cheng, J., Hao, J., Jiang, X., Ji, J., Wu, T., Chen, X., et al. (2021). Ameliorative Effects of miR-423-5p against Polarization of Microglial Cells of the M1 Phenotype by Targeting a NLRP3 Inflammasome Signaling Pathway. Int. Immunopharmacology 99, 108006. doi:10.1016/j.intimp.2021.108006

Chevriaux, A., Pilot, T., Derangère, V., Simonin, H., Martine, P., Chalmin, F., et al. (2020). Cathepsin B Is Required for NLRP3 Inflammasome Activation in Macrophages, through NLRP3 Interaction. Front. Cel Dev. Biol. 8, 167. doi:10.3389/fcell.2020.00167
Coll, R. C., Robertson, A. A. B., Chae, J. J., Higgins, S. C., Muñoz-Planillo, R., Inserra, M. C., et al. (2015). A Small-Molecule Inhibitor of the NLRP3 Inflammasome for the Treatment of Inflammatory Diseases. Nat. Med. 21 (3), 248-255. doi:10.1038/nm.3806

Cookson, B. T., and Brennan, M. A. (2001). Pro-inflammatory Programmed Cell Death. Trends Microbiol. 9 (3), 113-114. doi:10.1016/s0966-842x(00)01936-3

Darakhshan, S., and Pour, A. B. (2015). Tranilast: a Review of its Therapeutic Applications. Pharmacol. Res. 91, 15-28. doi:10.1016/j.phrs.2014.10.009

De Falco, G., Colarusso, C., Terlizzi, M., Popolo, A., Pecoraro, M., Commodo, M., et al. (2017). Chronic Obstructive Pulmonary Disease-Derived Circulating Cells Release IL-18 and IL-33 under Ultrafine Particulate Matter Exposure in a Caspase-1/8-independent Manner. Front. Immunol. 8, 1415. doi:10.3389/ fimmu.2017.01415

de Rivero Vaccari, J. P., Lotocki, G., Alonso, O. F., Bramlett, H. M., Dietrich, W. D., and Keane, R. W. (2009). Therapeutic Neutralization of the NLRP1 Inflammasome Reduces the Innate Immune Response and Improves Histopathology after Traumatic Brain Injury. J. Cereb. Blood Flow Metab. 29 (7), 1251-1261. doi:10.1038/jcbfm.2009.46

Demirel, I., Persson, A., Brauner, A., Särndahl, E., Kruse, R., and Persson, K. (2020). Activation of NLRP3 by Uropathogenic Escherichia coli Is Associated with IL$1 \beta$ Release and Regulation of Antimicrobial Properties in Human Neutrophils. Sci. Rep. 10 (1), 21837. doi:10.1038/s41598-020-78651-1

Dempsey, C., Rubio Araiz, A., Bryson, K. J., Finucane, O., Larkin, C., Mills, E. L., et al. (2017). Inhibiting the NLRP3 Inflammasome with MCC950 Promotes Non-phlogistic Clearance of Amyloid- $\beta$ and Cognitive Function in APP/PS1 Mice. Brain Behav. Immun. 61, 306-316. doi:10.1016/j.bbi.2016.12.014

Duncan, J. A., Bergstralh, D. T., Wang, Y., Willingham, S. B., Ye, Z., Zimmermann, A. G., et al. (2007). Cryopyrin/NALP3 Binds ATP/dATP, Is an ATPase, and Requires ATP Binding to Mediate Inflammatory Signaling. Proc. Natl. Acad. Sci. 104 (19), 8041-8046. doi:10.1073/pnas.0611496104

Ellis, A., Grace, P. M., Wieseler, J., Favret, J., Springer, K., Skarda, B., et al. (2016) Morphine Amplifies Mechanical Allodynia via TLR4 in a Rat Model of Spinal Cord Injury. Brain Behav. Immun. 58, 348-356. doi:10.1016/j.bbi.2016.08.004

Fan, X., Ma, W., Zhang, Y., and Zhang, L. (2020). P2X7 Receptor (P2X7R) of Microglia Mediates Neuroinflammation by Regulating (NOD)-Like Receptor Protein 3 (NLRP3) Inflammasome-dependent Inflammation after Spinal Cord Injury. Med. Sci. Monit. 26, e925491. doi:10.12659/MSM.925491

Fernandes-Alnemri, T., Kang, S., Anderson, C., Sagara, J., Fitzgerald, K. A., and Alnemri, E. S. (2013). Cutting Edge: TLR Signaling Licenses IRAK1 for Rapid Activation of the NLRP3 Inflammasome. J. Immunol. 191 (8), 3995-3999. doi:10.4049/jimmunol.1301681

Fernández-López, B., Valle-Maroto, S. M., Barreiro-Iglesias, A., and Rodicio, M. C. (2014). Neuronal Release and Successful Astrocyte Uptake of Aminoacidergic Neurotransmitters after Spinal Cord Injury in Lampreys. Glia 62 (8), 1254-1269. doi:10.1002/glia.22678

Ferreri, A. J., Illerhaus, G., Zucca, E., and Cavalli, F.International Extranodal Lymphoma Study, G. (2010). Flows and Flaws in Primary central Nervous System Lymphoma. Nat. Rev. Clin. Oncol. 7 (8), 1-2. doi:10.1038/ nrclinonc.2010.9-c1

Ge, X., Li, W., Huang, S., Yin, Z., Xu, X., Chen, F., et al. (2018). The Pathological Role of NLRs and AIM2 Inflammasome-Mediated Pyroptosis in Damaged Blood-Brain Barrier after Traumatic Brain Injury. Brain Res. 1697, 10-20. doi:10.1016/j.brainres.2018.06.008

Gordon, R., Albornoz, E. A., Christie, D. C., Langley, M. R., Kumar, V., Mantovani, S., et al. (2018). Inflammasome Inhibition Prevents $\alpha$-synuclein Pathology and Dopaminergic Neurodegeneration in Mice. Sci. Transl. Med. 10 (465), eaah4066. doi:10.1126/scitranslmed.aah4066

Gross, C. J., Mishra, R., Schneider, K. S., Médard, G., Wettmarshausen, J., Dittlein, D. C., et al. (2016). K + Efflux-independent NLRP3 Inflammasome Activation by Small Molecules Targeting Mitochondria. Immunity 45 (4), 761-773. doi:10.1016/j.immuni.2016.08.010

Gurung, P., Anand, P. K., Malireddi, R. K. S., Vande Walle, L., Van Opdenbosch, N., Dillon, C. P., et al. (2014). FADD and Caspase-8 Mediate Priming and Activation of the Canonical and Noncanonical Nlrp3 Inflammasomes. J. Immunol. 192 (4), 1835-1846. doi:10.4049/jimmunol.1302839

Halle, A., Hornung, V., Petzold, G. C., Stewart, C. R., Monks, B. G., Reinheckel, T., et al. (2008). The NALP3 Inflammasome Is Involved in the Innate Immune Response to Amyloid- $\beta$. Nat. Immunol. 9 (8), 857-865. doi:10.1038/ni.1636 
Hanada, M., Tsutsumi, K., Arima, H., Shinjo, R., Sugiura, Y., Imagama, S., et al. (2014). Evaluation of the Effect of Tranilast on Rats with Spinal Cord Injury. J. Neurol. Sci. 346 (1-2), 209-215. doi:10.1016/j.jns.2014.08.028

Hari, A., Zhang, Y., Tu, Z., Detampel, P., Stenner, M., Ganguly, A., et al. (2014). Activation of NLRP3 Inflammasome by Crystalline Structures via Cell Surface Contact. Sci. Rep. 4, 7281. doi:10.1038/srep07281

Harraz, M. M., Eacker, S. M., Wang, X., Dawson, T. M., and Dawson, V. L. (2012). MicroRNA-223 Is Neuroprotective by Targeting Glutamate Receptors. Proc. Natl. Acad. Sci. 109 (46), 18962-18967. doi:10.1073/pnas.1121288109

He, H., Jiang, H., Chen, Y., Ye, J., Wang, A., Wang, C., et al. (2018). Oridonin Is a Covalent NLRP3 Inhibitor with strong Anti-inflammasome Activity. Nat. Commun. 9 (1), 2550. doi:10.1038/s41467-018-04947-6

He, N., Zheng, X., He, T., Shen, G., Wang, K., Hu, J., et al. (2021a). MCC950 Reduces Neuronal Apoptosis in Spinal Cord Injury in Mice. Cnsnddt 20, 298-308. doi:10.2174/1871527319666201005170659

He, S.-D., Huang, S.-G., Zhu, H.-J., Luo, X.-G., Liao, K.-H., Zhang, J.-Y., et al. (2020b). Oridonin Suppresses Autophagy and Survival in Rheumatoid Arthritis Fibroblast-like Synoviocytes. Pharm. Biol. 58 (1), 146-151. doi:10.1080/ 13880209.2020 .1711783

He, X.-m., Zhou, Y.-z., Sheng, S., Li, J.-j., Wang, G.-q., and Zhang, F. (2020c). Ellagic Acid Protects Dopamine Neurons via Inhibition of NLRP3 Inflammasome Activation in Microglia. Oxidative Med. Cell Longevity 2020, 1-13. doi:10.1155/2020/2963540

Helmy, A., Carpenter, K. L., Menon, D. K., Pickard, J. D., and Hutchinson, P. J. (2011). The Cytokine Response to Human Traumatic Brain Injury: Temporal Profiles and Evidence for Cerebral Parenchymal Production. J. Cereb. Blood Flow Metab. 31 (2), 658-670. doi:10.1038/jcbfm.2010.142

Horng, T. (2014). Calcium Signaling and Mitochondrial Destabilization in the Triggering of the NLRP3 Inflammasome. Trends Immunol. 35 (6), 253-261. doi:10.1016/j.it.2014.02.007

Huang, Y., Jiang, H., Chen, Y., Wang, X., Yang, Y., Tao, J., et al. (2018). Tranilast Directly Targets NLRP 3 to Treat Inflammasome-driven Diseases. EMBO Mol. Med. 10 (4), e8689. doi:10.15252/emmm.201708689

Huang, Y., Wang, S., Huang, F., Zhang, Q., Qin, B., Liao, L., et al. (2021). c-FLIP Regulates Pyroptosis in Retinal Neurons Following Oxygen-Glucose Deprivation/recovery via a GSDMD-Mediated Pathway. Ann. Anat. Anatomischer Anzeiger 235, 151672. doi:10.1016/j.aanat.2020.151672

Ippagunta, S. K., Malireddi, R. K. S., Shaw, P. J., Neale, G. A., Walle, L. V., Green, D. R., et al. (2011). The Inflammasome Adaptor ASC Regulates the Function of Adaptive Immune Cells by Controlling Dock2-Mediated Rac Activation and Actin Polymerization. Nat. Immunol. 12 (10), 1010-1016. doi:10.1038/ni.2095

Irrera, N., Pizzino, G., Calò, M., Pallio, G., Mannino, F., Famà, F., et al. (2017). Lack of the Nlrp3 Inflammasome Improves Mice Recovery Following Traumatic Brain Injury. Front. Pharmacol. 8, 459. doi:10.3389/fphar.2017.00459

Ismael, S., Nasoohi, S., and Ishrat, T. (2018). MCC950, the Selective Inhibitor of Nucleotide Oligomerization Domain-like Receptor Protein-3 Inflammasome, Protects Mice against Traumatic Brain Injury. J. Neurotrauma 35 (11), 1294-1303. doi:10.1089/neu.2017.5344

Iyer, S. S., He, Q., Janczy, J. R., Elliott, E. I., Zhong, Z., Olivier, A. K., et al. (2013). Mitochondrial Cardiolipin Is Required for Nlrp3 Inflammasome Activation. Immunity 39 (2), 311-323. doi:10.1016/j.immuni.2013.08.001

Jayakumar, A. R., Tong, X. Y., Ruiz-Cordero, R., Bregy, A., Bethea, J. R., Bramlett, H. M., et al. (2014). Activation of NF-Kb Mediates Astrocyte Swelling and Brain Edema in Traumatic Brain Injury. J. Neurotrauma 31 (14), 1249-1257. doi:10.1089/neu.2013.3169

Jesenberger, V., Procyk, K. J., Yuan, J., Reipert, S., and Baccarini, M. (2000). Salmonella-Induced Caspase-2 Activation in Macrophages. J. Exp. Med. 192 (7), 1035-1046. doi:10.1084/jem.192.7.1035

Jia, Y.-J., Zhou, Y., Wen, L.-L., Li, Y.-F., Wu, K.-M., Duan, R.-R., et al. (2022). Exosomes Derived from Bone Marrow Mesenchymal Stem Cells Protect the Injured Spinal Cord by Inhibiting Pericyte Pyroptosis. Neural Regen. Res. 17 (1), 194-202. doi:10.4103/1673-5374.314323

Jiang, H., He, H., Chen, Y., Huang, W., Cheng, J., Ye, J., et al. (2017a). Identification of a Selective and Direct NLRP3 Inhibitor to Treat Inflammatory Disorders. J. Exp. Med. 214 (11), 3219-3238. doi:10.1084/jem.20171419

Jiang, W., Li, M., He, F., Zhou, S., and Zhu, L. (2017b). Targeting the NLRP3 Inflammasome to Attenuate Spinal Cord Injury in Mice. J. Neuroinflammation 14 (1), 207. doi:10.1186/s12974-017-0980-9
Jiang, W., Li, M., He, F., and Zhu, L. (2019). Inhibition of NLRP3 Inflammasome Attenuates Spinal Cord Injury-induced Lung Injury in Mice. J. Cel Physiol 234 (5), 6012-6022. doi:10.1002/jcp.27233

Jiao, J., Zhao, G., Wang, Y., Ren, P., and Wu, M. (2020). MCC950, a Selective Inhibitor of NLRP3 Inflammasome, Reduces the Inflammatory Response and Improves Neurological Outcomes in Mice Model of Spinal Cord Injury. Front. Mol. Biosci. 7, 37. doi:10.3389/fmolb.2020.00037

Jing, Y.-Y., Han, Z.-P., Sun, K., Zhang, S.-S., Hou, J., Liu, Y., et al. (2012). Toll-like Receptor 4 Signaling Promotes Epithelial-Mesenchymal Transition in Human Hepatocellular Carcinoma Induced by Lipopolysaccharide. BMC Med. 10, 98. doi:10.1186/1741-7015-10-98

Juliana, C., Fernandes-Alnemri, T., Kang, S., Farias, A., Qin, F., and Alnemri, E. S. (2012). Non-transcriptional Priming and Deubiquitination Regulate NLRP3 Inflammasome Activation. J. Biol. Chem. 287 (43), 36617-36622. doi:10.1074/ jbc.M112.407130

Juliana, C., Fernandes-Alnemri, T., Wu, J., Datta, P., Solorzano, L., Yu, J.-W., et al. (2010). Anti-inflammatory Compounds Parthenolide and Bay 11-7082 Are Direct Inhibitors of the Inflammasome. J. Biol. Chem. 285 (13), 9792-9802. doi:10.1074/jbc.M109.082305

Katsnelson, M. A., Rucker, L. G., Russo, H. M., and Dubyak, G. R. (2015). K+ Efflux Agonists Induce NLRP3 Inflammasome Activation Independently of $\mathrm{Ca} 2+$ Signaling. J.I. 194 (8), 3937-3952. doi:10.4049/jimmunol.1402658

Keller, S. A., Hernandez-Hopkins, D., Vider, J., Ponomarev, V., Hyjek, E., Schattner, E. J., et al. (2006). NF- $\mathrm{kB}$ Is Essential for the Progression of KSHV- and EBV-Infected Lymphomas In Vivo. Blood 107 (8), 3295-3302. doi:10.1182/blood-2005-07-2730

Kerr, N., de Rivero Vaccari, J., Umland, O., Bullock, M., Conner, G., Dietrich, W., et al. (2019). Human Lung Cell Pyroptosis Following Traumatic Brain Injury. Cells 8 (1), 69. doi:10.3390/cells8010069

Kerr, N., Lee, S. W., Perez-Barcena, J., Crespi, C., Ibañez, J., Bullock, M. R., et al. (2018). Inflammasome Proteins as Biomarkers of Traumatic Brain Injury. PLoS One 13 (12), e0210128. doi:10.1371/journal.pone.0210128

Kim, M.-J., Bae, S. H., Ryu, J.-C., Kwon, Y., Oh, J.-H., Kwon, J., et al. (2016). SESN2/ sestrin2 Suppresses Sepsis by Inducing Mitophagy and Inhibiting NLRP3 Activation in Macrophages. Autophagy 12 (8), 1272-1291. doi:10.1080/ 15548627.2016.1183081

Kim, T.-H., Yang, K., Kim, M., Kim, H.-S., and Kang, J. L. (2021). Apoptosis Inhibitor of Macrophage (AIM) Contributes to IL-10-induced Antiinflammatory Response through Inhibition of Inflammasome Activation. Cel Death Dis 12 (1), 19. doi:10.1038/s41419-020-03332-w

Kopp, R., Krautloher, A., Ramírez-Fernández, A., and Nicke, A. (2019). P2X7 Interactions and Signaling - Making Head or Tail of it. Front. Mol. Neurosci. 12, 183. doi:10.3389/fnmol.2019.00183

Kuang, N., Wang, X., Chen, Y., Liu, G., Kong, F., Wang, N., et al. (2021). Olfactory Ensheathing Cell Transplantation for Chronic Spinal Cord Injury: A Long-Term Follow-Up Study. J. Neurorestoratology 9 (2), 94-105. doi:10.26599/jnr.2021.9040008

Lamkanfi, M., and Dixit, V. M. (2014). Mechanisms and Functions of Inflammasomes. Cell 157 (5), 1013-1022. doi:10.1016/j.cell.2014.04.007

Lee, G.-S., Subramanian, N., Kim, A. I., Aksentijevich, I., Goldbach-Mansky, R., Sacks, D. B., et al. (2012). The Calcium-Sensing Receptor Regulates the NLRP3 Inflammasome through Ca2+ and cAMP. Nature 492 (7427), 123-127. doi:10.1038/nature11588

Lee, S. W., Gajavelli, S., Spurlock, M. S., Andreoni, C., de Rivero Vaccari, J. P., Bullock, M. R., et al. (2018). Microglial Inflammasome Activation in Penetrating Ballistic-like Brain Injury. J. Neurotrauma 35 (14), 1681-1693. doi:10.1089/neu.2017.5530

Li, C., Chen, M., He, X., and Ouyang, D. (2021). A Mini-Review on Ion Fluxes that Regulate NLRP3 Inflammasome Activation. Acta Biochim. Biophys. Sin (Shanghai) 53 (2), 131-139. doi:10.1093/abbs/gmaa155

Li, S., Li, H., Zhang, Y.-L., Xin, Q.-L., Guan, Z.-Q., Chen, X., et al. (2020a). SFTSV Infection Induces BAK/BAX-Dependent Mitochondrial DNA Release to Trigger NLRP3 Inflammasome Activation. Cel Rep. 30 (13), 4370-4385. doi:10.1016/j.celrep.2020.02.105

Li, X., Lou, X., Xu, S., Du, J., and Wu, J. (2020b). Hypoxia Inducible Factor-1 (HIF1a) Reduced Inflammation in Spinal Cord Injury via miR-380-3p/NLRP3 by Circ 0001723. Biol. Res. 53 (1), 35. doi:10.1186/s40659-020-00302-6

Li, X., Yu, Z., Zong, W., Chen, P., Li, J., Wang, M., et al. (2020c). Deficiency of the Microglial Hv1 Proton Channel Attenuates Neuronal Pyroptosis and Inhibits 
Inflammatory Reaction after Spinal Cord Injury. J. Neuroinflammation 17 (1), 263. doi:10.1186/s12974-020-01942-x

Lima, F. D., Souza, M. A., Furian, A. F., Rambo, L. M., Ribeiro, L. R., Martignoni, F. V., et al. (2008). Na+,K+-ATPase Activity Impairment after Experimental Traumatic Brain Injury: Relationship to Spatial Learning Deficits and Oxidative Stress. Behav. Brain Res. 193 (2), 306-310. doi:10.1016/ j.bbr.2008.05.013

Lin, K.-M., Hu, W., Troutman, T. D., Jennings, M., Brewer, T., Li, X., et al. (2014). IRAK-1 Bypasses Priming and Directly Links TLRs to Rapid NLRP3 Inflammasome Activation. Proc. Natl. Acad. Sci. 111 (2), 775-780. doi:10.1073/pnas.1320294111

Liu, H.-D., Li, W., Chen, Z.-R., Hu, Y.-C., Zhang, D.-D., Shen, W., et al. (2013). Expression of the NLRP3 Inflammasome in Cerebral Cortex after Traumatic Brain Injury in a Rat Model. Neurochem. Res. 38 (10), 2072-2083. doi:10.1007/ s11064-013-1115-Z

Liu, J., Zhu, Z., Wang, L., Du, J., Zhang, B., Feng, X., et al. (2020a). Functional Suppression of Ripk1 Blocks the NF-Kb Signaling Pathway and Induces Neuron Autophagy after Traumatic Brain Injury. Mol. Cel Biochem 472 (1-2), 105-114. doi:10.1007/s11010-020-03789-5

Liu, Q. Q., Wang, H. L., Chen, K., Wang, S. B., Xu, Y., Ye, Q., et al. (2016). Oridonin Derivative Ameliorates Experimental Colitis by Inhibiting Activated T-Cells and Translocation of Nuclear Factor-Kappa B. J. Dig. Dis. 17 (2), 104-112. doi:10.1111/1751-2980.12314

Liu, Z., Yao, X., Jiang, W., Li, W., Zhu, S., Liao, C., et al. (2020b). Advanced Oxidation Protein Products Induce Microglia-Mediated Neuroinflammation via MAPKs-NF-Kb Signaling Pathway and Pyroptosis after Secondary Spinal Cord Injury. J. Neuroinflammation 17 (1), 90. doi:10.1186/s12974-020-01751-2

Liu, Z., Yao, X., Sun, B., Jiang, W., Liao, C., Dai, X., et al. (2021). Pretreatment with Kaempferol Attenuates Microglia-Mediate Neuroinflammation by Inhibiting MAPKs-NF-Kb Signaling Pathway and Pyroptosis after Secondary Spinal Cord Injury. Free Radic. Biol. Med. 168, 142-154. doi:10.1016/ j.freeradbiomed.2021.03.037

Lu, A., Magupalli, V. G., Ruan, J., Yin, Q., Atianand, M. K., Vos, M. R., et al. (2014). Unified Polymerization Mechanism for the Assembly of ASC-dependent Inflammasomes. Cell 156 (6), 1193-1206. doi:10.1016/j.cell.2014.02.008

Lu, J., Chen, C., Deng, X., Mak, M. S., Zhu, Z., He, X., et al. (2020). Design, Synthesis, and Biological Evaluation of Novel Multifunctional RolipramTranilast Hybrids as Potential Treatment for Traumatic Brain Injury. ACS Chem. Neurosci. 11 (15), 2348-2360. doi:10.1021/acschemneuro.0c00339

Lv, Y., Sun, B., Lu, X.-X., Liu, Y.-l., Li, M., Xu, L.-X., et al. (2020). The Role of Microglia Mediated Pyroptosis in Neonatal Hypoxic-Ischemic Brain Damage. Biochem. Biophysical Res. Commun. 521 (4), 933-938. doi:10.1016/ j.bbrc.2019.11.003

Ma, C., Zhang, P., and Shen, Y. (2019). Progress in Research into Spinal Cord Injury Repair: Tissue Engineering Scaffolds and Cell Transdifferentiation. J. Neurorestoratology 7 (4), 196-206. doi:10.26599/jnr.2019.9040024

Ma, J., Xiao, W., Wang, J., Wu, J., Ren, J., Hou, J., et al. (2016). Propofol Inhibits NLRP3 Inflammasome and Attenuates Blast-Induced Traumatic Brain Injury in Rats. Inflammation 39 (6), 2094-2103. doi:10.1007/s10753-016-0446-8

Ma, M. W., Wang, J., Dhandapani, K. M., and Brann, D. W. (2017). NADPH Oxidase 2 Regulates NLRP3 Inflammasome Activation in the Brain after Traumatic Brain Injury. Oxidative Med. Cell Longevity 2017, 1-18. doi:10.1155/2017/6057609

Ma, Y., Jiang, J., Gao, Y., Shi, T., Zhu, X., Zhang, K., et al. (2018). Research Progress of the Relationship between Pyroptosis and Disease. Am. J. Transl Res. 10 (7), 2213-2219.

Mamik, M. K., and Power, C. (2017). Inflammasomes in Neurological Diseases: Emerging Pathogenic and Therapeutic Concepts. Brain 140 (9), 2273-2285. doi:10.1093/brain/awx133

Mangan, M. S. J., Olhava, E. J., Roush, W. R., Seidel, H. M., Glick, G. D., and Latz, E. (2018). Targeting the NLRP3 Inflammasome in Inflammatory Diseases. Nat. Rev. Drug Discov. 17 (8), 588-606. doi:10.1038/nrd.2018.97

Mantel, C., Messina-Graham, S., Moh, A., Cooper, S., Hangoc, G., Fu, X.-Y., et al. (2012). Mouse Hematopoietic Cell-Targeted STAT3 Deletion: Stem/progenitor Cell Defects, Mitochondrial Dysfunction, ROS Overproduction, and a Rapid Aging-like Phenotype. Blood 120 (13), 2589-2599. doi:10.1182/blood-2012-01404004
Mao, K., Chen, S., Chen, M., Ma, Y., Wang, Y., Huang, B., et al. (2013). Nitric Oxide Suppresses NLRP3 Inflammasome Activation and Protects against LPSInduced Septic Shock. Cell Res 23 (2), 201-212. doi:10.1038/cr.2013.6

Mariathasan, S., Weiss, D. S., Newton, K., McBride, J., O'Rourke, K., Roose-Girma, M., et al. (2006). Cryopyrin Activates the Inflammasome in Response to Toxins and ATP. Nature 440 (7081), 228-232. doi:10.1038/nature04515

Marongiu, L., Gornati, L., Artuso, I., Zanoni, I., and Granucci, F. (2019). Below the Surface: The Inner Lives of TLR4 and TLR9. J. Leukoc. Biol. 106 (1), 147-160. doi:10.1002/JLB.3MIR1218-483RR

Martinet, W., Coornaert, I., Puylaert, P., and De Meyer, G. R. Y. (2019). Macrophage Death as a Pharmacological Target in Atherosclerosis. Front. Pharmacol. 10, 306. doi:10.3389/fphar.2019.00306

Martinon, F., Pétrilli, V., Mayor, A., Tardivel, A., and Tschopp, J. (2006). Goutassociated Uric Acid Crystals Activate the NALP3 Inflammasome. Nature 440 (7081), 237-241. doi:10.1038/nature04516

Mbori, N. J. R., Chuan, X. Y., Feng, Q. X., Alizada, M., and Zhan, J. (2016). Evaluation of the Combination of Methylprednisolone and Tranilast after Spinal Cord Injury in Rat Models. J. Korean Neurosurg. Soc. 59 (4), 334-340. doi:10.3340/jkns.2016.59.4.334

McHugh, B. J., Wang, R., Li, H.-N., Beaumont, P. E., Kells, R., Stevens, H., et al. (2019). Cathelicidin Is a "fire Alarm", Generating Protective NLRP3-dependent Airway Epithelial Cell Inflammatory Responses during Infection with Pseudomonas aeruginosa. Plos Pathog. 15 (4), e1007694. doi:10.1371/ journal.ppat.1007694

Meng, J., Ding, T., Chen, Y., Long, T., Xu, Q., Lian, W., et al. (2021). LncRNA-Meg3 Promotes Nlrp3-Mediated Microglial Inflammation by Targeting miR-7a-5p. Int. Immunopharmacology 90, 107141. doi:10.1016/j.intimp.2020.107141

Miyake, T., Nakagami, H., and Morishita, R. (2012). Nucleic Acid Drugs for Preventing Restenosis after Coronary Revascularization. Ctmc 12 (15), 1613-1620. doi:10.2174/156802612803531324

Morquette, B., Juźwik, C. A., Drake, S. S., Charabati, M., Zhang, Y., Lécuyer, M.-A., et al. (2019). MicroRNA-223 Protects Neurons from Degeneration in Experimental Autoimmune Encephalomyelitis. Brain 142 (10), 2979-2995. doi:10.1093/brain/awz245

Mortezaee, K., Khanlarkhani, N., Beyer, C., and Zendedel, A. (2018). Inflammasome: Its Role in Traumatic Brain and Spinal Cord Injury. J. Cel Physiol 233 (7), 5160-5169. doi:10.1002/jcp.26287

Muñoz-Planillo, R., Kuffa, P., Martínez-Colón, G., Smith, B. L., Rajendiran, T. M., and Núñez, G. (2013). K+ Efflux Is the Common Trigger of NLRP3 Inflammasome Activation by Bacterial Toxins and Particulate Matter Immunity 38 (6), 1142-1153. doi:10.1016/j.immuni.2013.05.016

Murakami, T., Ockinger, J., Yu, J., Byles, V., McColl, A., Hofer, A. M., et al. (2012). Critical Role for Calcium Mobilization in Activation of the NLRP3 Inflammasome. Proc. Natl. Acad. Sci. 109 (28), 11282-11287. doi:10.1073/ pnas. 1117765109

Nakahira, K., Haspel, J. A., Rathinam, V. A. K., Lee, S.-J., Dolinay, T., Lam, H. C., et al. (2011). Autophagy Proteins Regulate Innate Immune Responses by Inhibiting the Release of Mitochondrial DNA Mediated by the NALP3 Inflammasome. Nat. Immunol. 12 (3), 222-230. doi:10.1038/ni.1980

Orning, P., Weng, D., Starheim, K., Ratner, D., Best, Z., Lee, B., et al. (2018). Pathogen Blockade of TAK1 Triggers Caspase-8-dependent Cleavage of Gasdermin D and Cell Death. Science 362 (6418), 1064-1069. doi:10.1126/ science.aau 2818

Ousingsawat, J., Schreiber, R., and Kunzelmann, K. (2019). TMEM16F/Anoctamin 6 in Ferroptotic Cell Death. Cancers 11 (5), 625. doi:10.3390/cancers11050625

Patel, B., Zheleznova, N. N., Ray, S. C., Sun, J., Cowley, A. W., Jr., and O'Connor, P. M. (2019). Voltage Gated Proton Channels Modulate Mitochondrial Reactive Oxygen Species Production by Complex I in Renal Medullary Thick Ascending Limb. Redox Biol. 27, 101191. doi:10.1016/j.redox.2019.101191

Pecorelli, A., Cordone, V., Messano, N., Zhang, C., Falone, S., Amicarelli, F., et al. (2020). Altered Inflammasome Machinery as a Key Player in the Perpetuation of Rett Syndrome Oxinflammation. Redox Biol. 28, 101334. doi:10.1016/ j.redox.2019.101334

Pellerin, L., and Magistretti, P. J. (1994). Glutamate Uptake into Astrocytes Stimulates Aerobic Glycolysis: a Mechanism Coupling Neuronal Activity to Glucose Utilization. Proc. Natl. Acad. Sci. 91 (22), 10625-10629. doi:10.1073/ pnas.91.22.10625 
Peng, J., Yi, M.-H., Jeong, H., McEwan, P. P., Zheng, J., Wu, G., et al. (2021). The Voltage-Gated Proton Channel Hvl Promotes Microglia-Astrocyte Communication and Neuropathic Pain after Peripheral Nerve Injury. Mol. Brain 14 (1), 99. doi:10.1186/s13041-021-00812-8

Peng, L., Wen, L., Shi, Q.-F., Gao, F., Huang, B., Meng, J., et al. (2020). Scutellarin Ameliorates Pulmonary Fibrosis through Inhibiting NF-Kb/nlrp3-Mediated Epithelial-Mesenchymal Transition and Inflammation. Cel Death Dis 11 (11), 978. doi:10.1038/s41419-020-03178-2

Perregaux, D. G., McNiff, P., Laliberte, R., Hawryluk, N., Peurano, H., Stam, E., et al. (2001). Identification and Characterization of a Novel Class of Interleukin1 post-translational Processing Inhibitors. J. Pharmacol. Exp. Ther. 299 (1), 187-197.

Perregaux, D., and Gabel, C. A. (1994). Interleukin-1 Beta Maturation and Release in Response to ATP and Nigericin. Evidence that Potassium Depletion Mediated by These Agents Is a Necessary and Common Feature of Their Activity. J. Biol. Chem. 269 (21), 15195-15203. doi:10.1016/s0021-9258(17) 36591-2

Pétrilli, V., Papin, S., Dostert, C., Mayor, A., Martinon, F., and Tschopp, J. (2007). Activation of the NALP3 Inflammasome Is Triggered by Low Intracellular Potassium Concentration. Cell Death Differ 14 (9), 1583-1589. doi:10.1038/ sj.cdd.4402195

Py, B. F., Kim, M.-S., Vakifahmetoglu-Norberg, H., and Yuan, J. (2013). Deubiquitination of NLRP3 by BRCC3 Critically Regulates Inflammasome Activity. Mol. Cel 49 (2), 331-338. doi:10.1016/j.molcel.2012.11.009

Qi, X., Man, S. M., Malireddi, R. K. S., Karki, R., Lupfer, C., Gurung, P., et al. (2016). Cathepsin B Modulates Lysosomal Biogenesis and Host Defense against Francisella Novicida Infection. J. Exp. Med. 213 (10), 2081-2097. doi:10.1084/jem.20151938

Qian, H., Li, Q., and Shi, W. (2017). Hyperbaric Oxygen Alleviates the Activation of NLRP-3-Inflammasomes in Traumatic Brain Injury. Mol. Med. Rep. 16 (4), 3922-3928. doi:10.3892/mmr.2017.7079

Reed, S. G., Orr, M. T., and Fox, C. B. (2013). Key Roles of Adjuvants in Modern Vaccines. Nat. Med. 19 (12), 1597-1608. doi:10.1038/nm.3409

Saijo, K., Crotti, A., and Glass, C. K. (2013). Regulation of Microglia Activation and Deactivation by Nuclear Receptors. Glia 61 (1), 104-111. doi:10.1002/ glia.22423

Sanman, L. E., Qian, Y., Eisele, N. A., Ng, T. M., van der Linden, W. A., Monack, D. M., et al. (2016). Disruption of Glycolytic Flux Is a Signal for Inflammasome Signaling and Pyroptotic Cell Death. Elife 5, e13663. doi:10.7554/eLife.13663

Sarhan, J., Liu, B. C., Muendlein, H. I., Li, P., Nilson, R., Tang, A. Y., et al. (2018). Caspase-8 Induces Cleavage of Gasdermin D to Elicit Pyroptosis duringYersiniainfection. Proc. Natl. Acad. Sci. USA 115 (46), E10888-E10897. doi:10.1073/pnas.1809548115

Schmidt, F. I., Lu, A., Chen, J. W., Ruan, J., Tang, C., Wu, H., et al. (2016). A Single Domain Antibody Fragment that Recognizes the Adaptor ASC Defines the Role of ASC Domains in Inflammasome Assembly. J. Exp. Med. 213 (5), 771-790. doi:10.1084/jem. 20151790

Sciarretta, S., Zhai, P., Shao, D., Maejima, Y., Robbins, J., Volpe, M., et al. (2012). Rheb Is a Critical Regulator of Autophagy during Myocardial Ischemia. Circulation 125 (9), 1134-1146. doi:10.1161/CIRCULATIONAHA.111.078212

Seo, S.-U., Kamada, N., Muñoz-Planillo, R., Kim, Y.-G., Kim, D., Koizumi, Y., et al. (2015). Distinct Commensals Induce Interleukin-1 $\beta$ via NLRP3 Inflammasome in Inflammatory Monocytes to Promote Intestinal Inflammation in Response to Injury. Immunity 42 (4), 744-755. doi:10.1016/j.immuni.2015.03.004

Shen, K., Jiang, W., Zhang, C., Cai, L., Wang, Q., Yu, H., et al. (2021). Molecular Mechanism of a Specific NLRP3 Inhibitor to Alleviate Seizure Severity Induced by Pentylenetetrazole. Curr. Mol. Pharmacol. 14, 579-586. doi:10.2174/ 1874467213666200810140749

Shi, J., Zhao, Y., Wang, K., Shi, X., Wang, Y., Huang, H., et al. (2015). Cleavage of GSDMD by Inflammatory Caspases Determines Pyroptotic Cell Death. Nature 526 (7575), 660-665. doi:10.1038/nature15514

Shi, M., Zhang, P., Vora, S. M., and Wu, H. (2020). Higher-order Assemblies in Innate Immune and Inflammatory Signaling: A General Principle in Cell Biology. Curr. Opin. Cel Biol. 63, 194-203. doi:10.1016/j.ceb.2020.03.002

Shimada, K., Crother, T. R., Karlin, J., Dagvadorj, J., Chiba, N., Chen, S., et al. (2012). Oxidized Mitochondrial DNA Activates the NLRP3 Inflammasome during Apoptosis. Immunity 36 (3), 401-414. doi:10.1016/ j.immuni.2012.01.009
Shimokawa, H., Sunamura, S., and Satoh, K. (2016). RhoA/Rho-Kinase in the Cardiovascular System. Circ. Res. 118 (2), 352-366. doi:10.1161/ CIRCRESAHA.115.306532

Si, L., Wang, H., and Wang, L. (2020). Suppression of miR-193a Alleviates Neuroinflammation and Improves Neurological Function Recovery after Traumatic Brain Injury (TBI) in Mice. Biochem. Biophysical Res. Commun. 523 (2), 527-534. doi:10.1016/j.bbrc.2019.11.095

Song, N., Liu, Z.-S., Xue, W., Bai, Z.-F., Wang, Q.-Y., Dai, J., et al. (2017). NLRP3 Phosphorylation Is an Essential Priming Event for Inflammasome Activation. Mol. Cel 68 (1), 185-197. e186. doi:10.1016/j.molcel.2017.08.017

Sun, B., Ding, R., Yu, W., Wu, Y., Wang, B., and Li, Q. (2016). Advanced Oxidative Protein Products Induced Human Keratinocyte Apoptosis through the NOXMAPK Pathway. Apoptosis 21 (7), 825-835. doi:10.1007/s10495-016-1245-2

Surprenant, A., Rassendren, F., Kawashima, E., North, R. A., and Buell, G. (1996). The Cytolytic P 2Z Receptor for Extracellular ATP Identified as a P 2X Receptor (P2X 7 ). Science 272 (5262), 735-738. doi:10.1126/science.272.5262.735

Tang, T., Lang, X., Xu, C., Wang, X., Gong, T., Yang, Y., et al. (2017). CLICsdependent Chloride Efflux Is an Essential and Proximal Upstream Event for NLRP3 Inflammasome Activation. Nat. Commun. 8 (1), 202. doi:10.1038/ s41467-017-00227-x

Toklu, H. Z., Hakan, T., Bỉber, N., Solakoğlu, S., Öğünç, A. z. V., and Şener, G. (2009). The Protective Effect of Alpha Lipoic Acid against Traumatic Brain Injury in Rats. Free Radic. Res. 43 (7), 658-667. doi:10.1080/ 10715760902988843

Viganò, E., Diamond, C. E., Spreafico, R., Balachander, A., Sobota, R. M., and Mortellaro, A. (2015). Human Caspase-4 and Caspase-5 Regulate the One-step Non-canonical Inflammasome Activation in Monocytes. Nat. Commun. 6, 8761. doi:10.1038/ncomms9761

Walev, I., Reske, K., Palmer, M., Valeva, A., and Bhakdi, S. (1995). Potassiuminhibited Processing of IL-1 Beta in Human Monocytes. EMBO J. 14 (8), 1607-1614. doi:10.1002/j.1460-2075.1995.tb07149.x

Wallisch, J. S., Simon, D. W., Bayır, H., Bell, M. J., Kochanek, P. M., and Clark, R. S. B. (2017). Cerebrospinal Fluid NLRP3 Is Increased after Severe Traumatic Brain Injury in Infants and Children. Neurocrit. Care 27 (1), 44-50. doi:10.1007/s12028-017-0378-7

Wang, Y., Ni, H., Li, H., Deng, H., Xu, L. S., Xu, S., et al. (2018). Nuclear Factor Kappa B Regulated Monocyte Chemoattractant Protein-1/chemokine CC Motif Receptor-2 Expressing in Spinal Cord Contributes to the Maintenance of Cancer-Induced Bone Pain in Rats. Mol. Pain 14, 174480691878868. doi:10.1177/1744806918788681

Ward, R., Li, W., Abdul, Y., Jackson, L., Dong, G., Jamil, S., et al. (2019). NLRP3 Inflammasome Inhibition with MCC950 Improves Diabetes-Mediated Cognitive Impairment and Vasoneuronal Remodeling after Ischemia. Pharmacol. Res. 142, 237-250. doi:10.1016/j.phrs.2019.01.035

Weber, K., and Schilling, J. D. (2014). Lysosomes Integrate MetabolicInflammatory Cross-Talk in Primary Macrophage Inflammasome Activation. J. Biol. Chem. 289 (13), 9158-9171. doi:10.1074/jbc.M113.531202

Wei, X., Hu, C.-c., Zhang, Y.-1., Yao, S.-1., and Mao, W.-k. (2016). Telmisartan Reduced Cerebral Edema by Inhibiting NLRP3 Inflammasome in Mice with Cold Brain Injury. J. Huazhong Univ. Sci. Technol. [Med. Sci. 36 (4), 576-583. doi:10.1007/s11596-016-1628-1

Wolf, A. J., Reyes, C. N., Liang, W., Becker, C., Shimada, K., Wheeler, M. L., et al. (2016). Hexokinase Is an Innate Immune Receptor for the Detection of Bacterial Peptidoglycan. Cell 166 (3), 624-636. doi:10.1016/j.cell.2016.05.076

Wu, C., Chen, H., Zhuang, R., Zhang, H., Wang, Y., Hu, X., et al. (2021). Betulinic Acid Inhibits Pyroptosis in Spinal Cord Injury by Augmenting Autophagy via the AMPK-mTOR-TFEB Signaling Pathway. Int. J. Biol. Sci. 17 (4), 1138-1152. doi:10.7150/ijbs. 57825

$\mathrm{Wu}$, J., Fernandes-Alnemri, T., and Alnemri, E. S. (2010). Involvement of the AIM2, NLRC4, and NLRP3 Inflammasomes in Caspase-1 Activation by Listeria Monocytogenes. J. Clin. Immunol. 30 (5), 693-702. doi:10.1007/s10875-0109425-2

Xing, Y., Yao, X., Li, H., Xue, G., Guo, Q., Yang, G., et al. (2017). Cutting Edge: TRAF6 Mediates TLR/IL-1R Signaling-Induced Nontranscriptional Priming of the NLRP3 Inflammasome. J. Immunol. 199 (5), 1561-1566. doi:10.4049/ jimmunol.1700175

Xu, L., Wang, Q., Jiang, W., Yu, S., and Zhang, S. (2019). MiR-34c Ameliorates Neuropathic Pain by Targeting NLRP3 in a Mouse Model of Chronic 
Constriction Injury. Neuroscience 399, 125-134. doi:10.1016/ j.neuroscience.2018.12.030

Xu, S., Wang, J., Jiang, J., Song, J., Zhu, W., Zhang, F., et al. (2020). TLR4 Promotes Microglial Pyroptosis via IncRNA-F630028O10Rik by Activating PI3K/AKT Pathway after Spinal Cord Injury. Cel Death Dis 11 (8), 693. doi:10.1038/ s41419-020-02824-z

Xu, S., Wang, J., Zhong, J., Shao, M., Jiang, J., Song, J., et al. (2021). CD73 Alleviates GSDMD-mediated Microglia Pyroptosis in Spinal Cord Injury through PI3K/ AKT/Foxo1 Signaling. Clin. Translational Med. 11 (1), e269. doi:10.1002/ ctm2.269

Xu, X., Yin, D., Ren, H., Gao, W., Li, F., Sun, D., et al. (2018). Selective NLRP3 Inflammasome Inhibitor Reduces Neuroinflammation and Improves LongTerm Neurological Outcomes in a Murine Model of Traumatic Brain Injury. Neurobiol. Dis. 117, 15-27. doi:10.1016/j.nbd.2018.05.016

Yan, C., Yan, H., Mao, J., Liu, Y., Xu, L., Zhao, H., et al. (2020). Neuroprotective Effect of Oridonin on Traumatic Brain Injury via Inhibiting NLRP3 Inflammasome in Experimental Mice. Front. Neurosci. 14, 557170. doi:10.3389/fnins.2020.557170

Yang, Z., Zhong, L., Xian, R., and Yuan, B. (2015). MicroRNA-223 Regulates Inflammation and Brain Injury via Feedback to NLRP3 Inflammasome after Intracerebral Hemorrhage. Mol. Immunol. 65 (2), 267-276. doi:10.1016/ j.molimm.2014.12.018

Ye, B., Chen, X., Dai, S., Han, J., Liang, X., Lin, S., et al. (2019). Emodin Alleviates Myocardial Ischemia/reperfusion Injury by Inhibiting Gasdermin D-Mediated Pyroptosis in Cardiomyocytes. Drug Des. Develop. Ther. 13, 975-990. doi:10.2147/DDDT.S195412

Yi, J.-H., and Hazell, A. S. (2006). Excitotoxic Mechanisms and the Role of Astrocytic Glutamate Transporters in Traumatic Brain Injury. Neurochem. Int. 48 (5), 394-403. doi:10.1016/j.neuint.2005.12.001

Yi, Y.-S. (2020). Caspase-11 Non-canonical Inflammasome: Emerging Activator and Regulator of Infection-Mediated Inflammatory Responses. Int. J. Mol. Sci. 21 (8), 2736. doi:10.3390/ijms 21082736

Yu, T., Dohl, J., Elenberg, F., Chen, Y., and Deuster, P. (2019a). Curcumin Induces Concentration-dependent Alterations in Mitochondrial Function through ROS in C2C12 Mouse Myoblasts. J. Cel Physiol 234 (5), 6371-6381. doi:10.1002/jcp.27370

Yu, T., Xie, W., and Sun, Y. (2019b). Oridonin Inhibits LPS-Induced Inflammation in Human Gingival Fibroblasts by Activating PPAR $\gamma$. Int. Immunopharmacology 72, 301-307. doi:10.1016/j.intimp.2019.04.006

Zendedel, A., Johann, S., Mehrabi, S., Joghataei, M.-t., Hassanzadeh, G., Kipp, M., et al. (2016). Activation and Regulation of NLRP3 Inflammasome by Intrathecal Application of SDF-1a in a Spinal Cord Injury Model. Mol. Neurobiol. 53 (5), 3063-3075. doi:10.1007/s12035-015-9203-5

Zendedel, A., Mönnink, F., Hassanzadeh, G., Zaminy, A., Ansar, M. M., Habib, P., et al. (2018). Estrogen Attenuates Local Inflammasome Expression and Activation after Spinal Cord Injury. Mol. Neurobiol. 55 (2), 1364-1375. doi:10.1007/s12035-017-0400-2

Zhang, H.-B., Cheng, S.-X., Tu, Y., Zhang, S., Hou, S.-K., and Yang, Z. (2017). Protective Effect of Mild-Induced Hypothermia against Moderate Traumatic Brain Injury in Rats Involved in Necroptotic and Apoptotic Pathways. Brain Inj. 31 (3), 406-415. doi:10.1080/02699052.2016.1225984

Zhang, M., Wang, L., Huang, S., and He, X. (2020). MicroRNA-223 Targets NLRP3 to Relieve Inflammation and Alleviate Spinal Cord Injury. Life Sci. 254, 117796. doi:10.1016/j.lfs.2020.117796
Zhao, S., Li, X., Wang, J., and Wang, H. (2021). The Role of the Effects of Autophagy on NLRP3 Inflammasome in Inflammatory Nervous System Diseases. Front. Cel Dev. Biol. 9, 657478. doi:10.3389/fcell.2021.657478

Zheng, B., Zhang, S., Ying, Y., Guo, X., Li, H., Xu, L., et al. (2018a). Administration of Dexmedetomidine Inhibited NLRP3 Inflammasome and Microglial Cell Activities in hippocampus of Traumatic Brain Injury Rats. Biosci. Rep. 38 (5), BSR20180892. doi:10.1042/BSR20180892

Zheng, G., Zhan, Y., Wang, H., Luo, Z., Zheng, F., Zhou, Y., et al. (2019). Carbon Monoxide Releasing Molecule-3 Alleviates Neuron Death after Spinal Cord Injury via Inflammasome Regulation. EBioMedicine 40, 643-654. doi:10.1016/ j.ebiom.2018.12.059

Zheng, R., Tao, L., Jian, H., Chang, Y., Cheng, Y., Feng, Y., et al. (2018b). NLRP3 Inflammasome Activation and Lung Fibrosis Caused by Airborne fine Particulate Matter. Ecotoxicology Environ. Saf. 163, 612-619. doi:10.1016/ j.ecoenv.2018.07.076

Zhong, Y., Kinio, A., and Saleh, M. (2013). Functions of NOD-like Receptors in Human Diseases. Front. Immunol. 4, 333. doi:10.3389/fimmu.2013.00333

Zhong, Z., Liang, S., Sanchez-Lopez, E., He, F., Shalapour, S., Lin, X.-j., et al. (2018). New Mitochondrial DNA Synthesis Enables NLRP3 Inflammasome Activation. Nature 560 (7717), 198-203. doi:10.1038/s41586-018-0372-z

Zhou, K., Shi, L., Wang, Y., Chen, S., and Zhang, J. (2016). Recent Advances of the NLRP3 Inflammasome in Central Nervous System Disorders. J. Immunol. Res. 2016, 1-9. doi:10.1155/2016/9238290

Zhou, R., Yazdi, A. S., Menu, P., and Tschopp, J. (2011). A Role for Mitochondria in NLRP3 Inflammasome Activation. Nature 469 (7329), 221-225. doi:10.1038/ nature09663

Zhu, J., Yang, J., and Xu, J. (2021). miR-223 Inhibits the Polarization and Recruitment of Macrophages via NLRP3/IL-1 $\beta$ Pathway to Meliorate Neuropathic Pain. Pain Res. Manag. 2021, 1-11. doi:10.1155/2021/6674028

Zychlinsky, A., Prevost, M. C., and Sansonetti, P. J. (1992). Shigella Flexneri Induces Apoptosis in Infected Macrophages. Nature 358 (6382), 167-169. doi:10.1038/358167a0

Conflict of Interest: The authors declare that the research was conducted in the absence of any commercial or financial relationships that could be construed as a potential conflict of interest.

The handling Editor LW has declared a shared parent affiliation with the authors at the time of review.

Publisher's Note: All claims expressed in this article are solely those of the authors and do not necessarily represent those of their affiliated organizations, or those of the publisher, the editors and the reviewers. Any product that may be evaluated in this article, or claim that may be made by its manufacturer, is not guaranteed or endorsed by the publisher.

Copyright (c) 2022 Zhou, Zheng, Fan and Wu. This is an open-access article distributed under the terms of the Creative Commons Attribution License (CC BY). The use, distribution or reproduction in other forums is permitted, provided the original author(s) and the copyright owner(s) are credited and that the original publication in this journal is cited, in accordance with accepted academic practice. No use, distribution or reproduction is permitted which does not comply with these terms. 